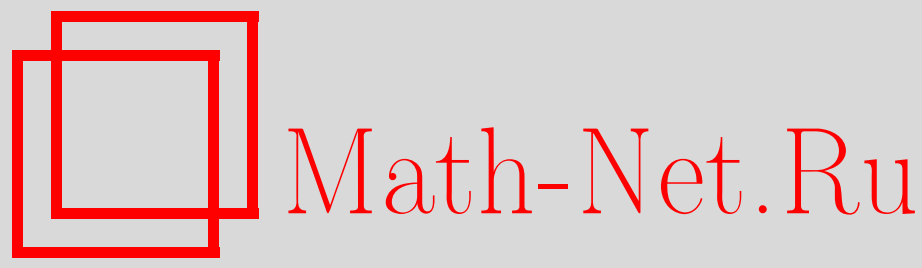

М. Бордаг, Вакуумная и тепловая энергии для двух осцилляторов, взаимодействующих посредством поля, ТМФ, 2018, том 195, номер 3, 391-421

DOI: https://doi.org/10.4213/tmf9407

Использование Общероссийского математического портала Math-Net.Ru подразумевает, что вы прочитали и согласны с пользовательским соглашением http://www . mathnet.ru/rus/agreement

Параметры загрузки:

IP : 3.85 .7 .115

26 апреля 2023 г., $18: 34: 16$

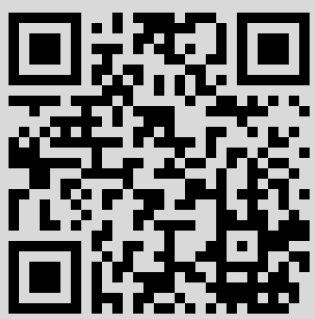




\title{
ВАКУУМНАЯ И ТЕПЛОВАЯ ЭНЕРГИИ ДЛЯ ДВУХ ОСЦИЛЛЯТОРОВ, ВЗАИМОДЕЙСТВУЮЩИХ ПОСРЕДСТВОМ ПОЛЯ
}

\begin{abstract}
Рассматривается простая $(1+1)$-модель взаимодействия Казимира-Полдера, которая включает в себя два осциллятора, связанных скалярным полем. Рассматривается диссипация в рамках подхода, основанного на первых принципах, при допущении, что осцилляторы взаимодействуют с термостатами. Для данной системы выведено уравнение для свободной энергии в терминах вещественных частот. На основе данного представления получено представление Мацубары для случая диссипации. Рассмотрен случай нулевых собственных частот осцилляторов. Показано, что в данном случае вклад от нулевой частоты Мацубары становится модифицированным и не возникает проблем, связанных с законами термодинамики.
\end{abstract}

Ключевые слова: сила Казимира-Полдера, температура, диссипация, резервуар.

DOI: https://doi.org/10.4213/tmf9407

\section{1. ВВЕДЕНИЕ}

Сила Казимира-Полдера описывает базовое электромагнитное взаимодействие между молекулами или между молекулой и стенкой, а формула Лифшица описывает взаимодействие между макроскопическими телами. Теоретически данный подход обычно основана на стохастической термодинамике и включает в себя рассмотрение свойств вещества с точки зрения проницаемости или поляризуемости. Таким образом, возможны учет реальной структуры взаимодействующих объектов и предсказание результатов измерений высокой точности, которые представляют собой весьма актуальную и важную проблему.

Наряду с очевидными достижениями в данной области существуют определенные неясности, связанные с диссипацией. В простейшем случае показано, что при введении проницаемости Друде в формулу Лифшица происходит нарушение термодинамического принципа [1], в другом случае при учете удельной электропроводности по постоянному току при рассмотрении взаимодействия между атомом и стенкой наблюдается расхождение с экспериментальными результатами [2]. Современное

*Institute of Theoretical Physics, Leipzig University, Leipzig, Germany. E-mail: bordag@uni-leipzig.de 
состояние исследований в данном направлении представлено в работе [3]. Следует также отметить проблему, связанную с тем, что только при использовании предписания Швингера фиксированная проницаемость при стремлении к бесконечности воспроизводит предел идеального проводника [4].

Следует отметить, что введение некоторой модели проницаемости или поляризуемости в случае сил Казимира-Полдера в формулу Лифшица представляет собой чисто феноменологический подход, а следовательно, требуется разработка более фундаментальной модели. Для решения данной проблемы обычно вводят связь осцилляторов, описывающих молекулы, с термостатом (резервуаром). Такой подход позволяет сформулировать основные принципы, начиная, например, с гамильтониана. Затем остается проблема разработки подхода для данной конфигурации. Для сил Казимира-Полдера и сил Казимира такого рода система состоит из диполей, взаимодействующих с электромагнитным полем и взаимодействующих с соответствующими термостатами. Следовательно, диссипация является результатом взаимодействия с термостатами и связана с силами Ланжевена.

В настоящее время разработке такого рода идей посвящено большое количество работ (см., например, [5]-[10]), включая работы, в которых рассматриваются силы Казимира-Полдера и силы Казимира. Более того, подход с учетом термостатов позволяет рассматривать неравновесные случаи, радиационный перенос тепла, а также широкий круг явлений, обычно встречающийся в открытых квантовых системах [11]. Однако в данных работах, в которых рассматривается случай однородной среды, авторы ограничиваются осцилляторами с ненулевой собственной частотой или осцилляторами, разнесенными на большое расстояние, и в основном воспроизводят известные результаты, касающиеся сил Казимира-Полдера, или формулы Лифшица.

Данная проблема может быть сформулирована в несколько ином ключе при рассмотрении частот. Известно, что в случае отсутствия диссипации рассматриваемая задача имеет вещественные собственные частоты, и термодинамические величины, такие как свободная энергия, могут быть представлены как сумма или интеграл по всем такого рода частотам. Эквивалентное представление в рамках мнимых частот Мацубары связано с поворотом Вика. В случае диссипации собственные частоты не являются действительными, а свободная энергия не может быть представлена в терминах собственных частот, но только в терминах частот Мацубары. Попытка наивно осуществить обратный поворот Вика не осуществима [12]. Показано [13], что представление в рамках комплексных частот (резонансов) возможно, но не считается особенно вдохновляющим.

В настоящей работе разработан фундаментальный подход для описания диссипации посредством связи термостатов для простейшей системы, имитирующей взаимодействие Казимира-Полдера. Рассмотрены два одномерных осциллятора, каждый из которых связан с термостатом, а оба осциллятора связаны с $(1+1)$-размерным скалярным полем. В данной работе не проведено разнесение на большое расстояние с самого начала, и, таким образом, рассмотрение включает непертурбативные эффекты, которые модифицируют представление Мацубары. Осцилляторы имеют собственную частоту $\Omega$, которая может быть сведена к нулю во время или по окончании вычислений. Свободная энергия системы выражена как сумма или интеграл по действительным частотам. Исследованы свойства данного представления, например показана связь с представлением Мацубары и рассмотрены границы низких температур, нулевая диссипация, а также нулевая собственная частота $\Omega$. 
Базовые идеи, касающиеся термостатов, которые использовались в настоящей статье, не являются новыми. Они в основном аналогичны идеям, представленным в знаменитой публикации Фано [14], в подходе Хаттнера-Барнетта [15], а также в подходе открытых квантовых систем [8]. Однако в контексте взаимодействий Казимира и Казимира-Полдера с диссипацией данные идеи широко используются лишь с недавнего времени. По этой причине в настоящей статье приведено самое простое решение, которое может быть легко использовано и которое является достаточным для описания всех характерных особенностей, которые представляют интерес. Более того, следует отметить, что подход с учетом термостатов может быть переформулирован для равновесного случая в рамках флуктуационно-диссипационной теоремы, предложенной в работе [16].

В разделе 2 рассматривается случай взаимодействия одиночного осциллятора с термостатом, в разделе 3 изучается случай взаимодействия двух осцилляторов со скалярным полем. В разделе 4 исследуется полная система в ящике конечного размера и выведено представление в рамках действительных частот. В разделе 5 рассмотрены особые случаи и переход к частотам Мацубары. В разделе 6 изучаются системы в неограниченном пространстве, заключительные выводы представлены в разделе 7.

В данной статье использованы единицы с $c=k_{\mathrm{B}}=1$.

\section{2. ОДИНОЧНЫЙ ОСЦИЛЛЯТОР, ВЗАИМОДЕЙСТВУЮЩИЙ С ТЕРМОСТАТОМ}

В данном разделе рассмотрим одиночный гармонический осциллятор, взаимодействующий с термостатом. Этот случай представляет собой простейшую термодинамическую систему, которая описана во многих учебниках. Приведем основные формулы для их дальнейшего использования при решении наших задач.

2.1. Основные формулы. Рассмотрим гармонический осциллятор $\xi(t)$, связанный с бесконечным набором осцилляторов термостатов $q_{\omega}(t)$. Данная система описывается следующим лагранжианом

$$
\mathcal{L}=\frac{m}{2}\left(\dot{\xi}(t)^{2}+\Omega^{2} \xi(t)^{2}\right)+\int_{0}^{\infty} d \omega \frac{\mu}{2}\left[\dot{q}_{\omega}(t)^{2}+\omega^{2}\left(q_{\omega}(t)-\xi(t)\right)^{2}\right],
$$

где $\mu$ - плотность массы термостатных осцилляторов. Выбор связи между осцилляторами, который следует работе [17], позволяет избежать искусственных расходимостей. В лагранжиане (1) все переменные имеют зависимость от времени, которую в дальнейшем для простоты изложения мы писать не будем. В соответствии с формулой (1) гамильтониан записывается следующим образом:

$$
H=H_{\mathrm{osc}}+H_{\mathrm{bath}}+H_{\mathrm{int}},
$$

где

$$
\begin{aligned}
H_{\mathrm{osc}} & =\frac{p^{2}}{2 m}+\frac{m \Omega^{2}}{2} \xi^{2}, \\
H_{\mathrm{bath}} & =\int_{0}^{\infty} d \omega\left(\frac{p_{\omega}^{2}}{2 \mu}+\frac{\mu \omega^{2}}{2} q_{\omega}^{2}\right), \\
H_{\mathrm{int}} & =\int_{0}^{\infty} d \omega \frac{\mu \omega^{2}}{2}\left(-2 q_{\omega}+\xi\right) \xi
\end{aligned}
$$


сопряженные моменты записываются как

$$
p=\frac{\partial \mathcal{L}}{\partial \dot{\xi}}, \quad p_{\omega}=\frac{\partial \mathcal{L}}{\partial \dot{q}_{\omega}} .
$$

Из лагранжиана (1) следуют уравнения движения

$$
m\left(\ddot{\xi}+\Omega^{2} \xi\right)=\int_{0}^{\infty} d \omega \mu \omega^{2}\left(q_{\omega}-\xi\right)
$$

для осциллятора и

$$
\mu\left(\ddot{q}_{\omega}+\omega^{2} q_{\omega}\right)=\mu \omega^{2} \xi
$$

для переменных термостата.

Теперь произведем квантование системы (1), (2). Введем операторы рождения и уничтожения $\hat{b}_{\omega}^{\dagger}$ и $\hat{b}_{\omega}$ термостата с коммутационным соотношением

$$
\left[\hat{b}_{\omega}, \hat{b}_{\omega^{\prime}}^{\dagger}\right]=\delta\left(\omega-\omega^{\prime}\right)
$$

и используем формулы

$$
\hat{q}_{\omega}=\frac{l_{0}}{\sqrt{2}}\left(\hat{b}_{\omega}+\hat{b}_{\omega}^{\dagger}\right), \quad \hat{p}_{\omega}=\frac{\hbar}{i \sqrt{2} l_{0}}\left(\hat{b}_{\omega}-\hat{b}_{\omega}^{\dagger}\right)
$$

для соответствующих операторов координат и импульса. В данном случае величина

$$
l_{0}=\sqrt{\frac{\hbar}{\mu \omega}}
$$

представляет собой характеристическую длину, связанную с осцилляторами термостата.

Оператор Гамильтона в соответствии с (4) представлен как

$$
\hat{H}_{\mathrm{bath}}=\int_{0}^{\infty} d \omega \frac{\hbar \omega}{2}\left(\hat{b}_{\omega}^{\dagger} \hat{b}_{\omega}+\hat{b}_{\omega} \hat{b}_{\omega}^{\dagger}\right)
$$

Далее рассмотрим уравнения движения Гейзенберга для операторов $\hat{b}_{\omega}^{\dagger}(t)$ и $\hat{b}_{\omega}(t)$ (в данном случае мы снова пишем зависимость от времени)

$$
\begin{aligned}
& \dot{\hat{b}}_{\omega}(t)=\frac{i}{\hbar}\left[\hat{H}, \hat{b}_{\omega}(t)\right]=-i \omega \hat{b}_{\omega}(t)-i \frac{\omega}{\sqrt{2} l_{0}} \hat{\xi}(t), \\
& \dot{\hat{b}}_{\omega}^{\dagger}(t)=\frac{i}{\hbar}\left[\hat{H}, \hat{b}_{\omega}^{\dagger}(t)\right]=i \omega \hat{b}_{\omega}^{\dagger}(t)+i \frac{\omega}{\sqrt{2} l_{0}} \hat{\xi}(t),
\end{aligned}
$$

где используется (11), и в соответствии с (10) получаем следующее уравнение для осциллятора:

$$
m\left(\ddot{\hat{\xi}}+\Omega^{2} \hat{\xi}\right)=\int_{0}^{\infty} d \omega \frac{\mu \omega^{2} l_{0}}{\sqrt{2}}\left(\hat{b}_{\omega}(t)+\hat{b}_{\omega}^{\dagger}(t)\right)-\int_{0}^{\infty} d \omega \mu \omega^{2} \hat{\xi}(t) .
$$

Решения уравнений (13) представляют собой операторы Гейзенберга,

$$
\begin{aligned}
& \hat{b}_{\omega}(t)=e^{-i \omega t} \hat{b}_{\omega}(0)+\frac{i \omega}{\sqrt{2} l_{0}} \int_{-\infty}^{t} d t^{\prime} e^{-i \omega\left(t-t^{\prime}\right)} \hat{\xi}\left(t^{\prime}\right), \\
& \hat{b}_{\omega}^{\dagger}(t)=e^{i \omega t} \hat{b}_{\omega}^{\dagger}(0)-\frac{i \omega}{\sqrt{2} l_{0}} \int_{-\infty}^{t} d t^{\prime} e^{i \omega\left(t-t^{\prime}\right)} \hat{\xi}\left(t^{\prime}\right),
\end{aligned}
$$


в предположении, что поле $\hat{\xi}(t)$ отсутствует при $t=-\infty$, а решение является запаздывающим. Операторы $\hat{b}_{\omega}^{\dagger}(0)$ и $\hat{b}_{\omega}(0)$ представляют собой начальные операторы, удовлетворяющие коммутационному соотношению (9).

Данные операторы позволяют записать оператор координаты для осцилляторов термостата также в представлении Гейзенберга, из (10) имеем

$$
\hat{q}_{\omega}(t)=\hat{q}_{\omega}^{\mathrm{h}}(t)+\int_{-\infty}^{t} d t^{\prime} \omega \sin \left(\omega\left(t-t^{\prime}\right)\right) \hat{\xi}\left(t^{\prime}\right)
$$

где однородное решение

$$
\hat{q}_{\omega}^{\mathrm{h}}(t)=\frac{l_{0}}{\sqrt{2}}\left(e^{-i \omega t} \hat{b}_{\omega}(0)+e^{i \omega t} \hat{b}_{\omega}^{\dagger}(0)\right)
$$

Подставив решение (15) в правую часть уравнения (14) для осциллятора и используя (17), получаем

$$
m\left(\partial_{t}^{2}+\Omega^{2}\right) \hat{\xi}(t)=F_{\mathrm{L}}(t)-\hat{\Gamma} \hat{\xi}(t)
$$

где

$$
F_{\mathrm{L}}(t)=\int_{0}^{\infty} d \omega \mu \omega^{2} \hat{q}_{\omega}^{\mathrm{h}}(t)
$$

представляет собой хорошо известную силу Ланжевена. С учетом (17) это выражение также может быть записано в следующем виде:

$$
F_{\mathrm{L}}(t)=\int_{0}^{\infty} d \omega \frac{\mu \omega^{2} l_{0}}{\sqrt{2}}\left(e^{-i \omega t} \hat{b}_{\omega}(0)+e^{i \omega t} \hat{b}_{\omega}^{\dagger}(0)\right) .
$$

Второй член в правой части (18) может быть представлен как

$$
-\hat{\Gamma} \hat{\xi}(t) \equiv \int_{0}^{\infty} d \omega \mu \omega^{2}\left[\int_{-\infty}^{t} d t^{\prime} \omega \sin \left(\omega\left(t-t^{\prime}\right)\right) \hat{\xi}\left(t^{\prime}\right)-\hat{\xi}(t)\right] .
$$

Здесь $\hat{\Gamma}$ обозначает линейный положительный оператор [17]. Обычно рассмотрение начинают с использования общих свойств, но в данном случае более конструктивным нам представляется подход с ограничением рассмотрения случаем линейного затухания при параметризации плотности массы $\mu$ термостата в виде

$$
\mu=\frac{2 \gamma m}{\pi \omega^{2}} \frac{1}{1+(\delta \omega)^{2}},
$$

где $\gamma$ - параметр диссипации, а $\delta$ - параметр регуляризации типа Друде с $\delta \rightarrow 0$ в конце вычислений.

Используя параметризацию (22), мы получаем

$$
-\hat{\Gamma} \hat{\xi}(t)=-\gamma m \dot{\hat{\xi}}(t)
$$

для линейного затухания. Подставляя этот член в левую часть уравнения (18), получаем уравнение

$$
m\left(\partial_{t}^{2}+\gamma \partial_{t}+\Omega^{2}\right) \hat{\xi}(t)=F_{\mathrm{L}}(t)
$$


для гармонического осциллятора $\hat{\xi}(t)$, который демпфируется параметром $\gamma$ и возбуждается силой Ланжевена $F_{\mathrm{L}}(t)$. Такого рода сценарий представляет собой хорошо известный подход к рассмотрению термостата, связанного с осциллятором. Термостат обеспечивает диссипацию $\gamma$ и в равновесии обеспечивает возмущения, которые поддерживают осциллятор в стационарном состоянии.

Уравнение (24) может быть решено самым простым способом, с использованием преобразования Фурье. В данном случае и далее для всех зависящих от времени величин мы примем следующие соглашения относительно преобразования Фурье:

$$
\xi(t)=\int_{-\infty}^{\infty} \frac{d \omega}{2 \pi} e^{i \omega t} \tilde{\xi}_{\omega}, \quad \tilde{\xi}_{\omega}=\int_{-\infty}^{\infty} d t e^{-i \omega t} \xi(t) .
$$

Тогда уравнение (24) преобразуется следующим образом:

$$
m N(\omega) \tilde{\xi}_{\omega}=\widetilde{F}_{\omega}
$$

где

$$
N(\omega) \equiv-\omega^{2}+i \gamma \omega+\Omega^{2}
$$

a

$$
\widetilde{F}_{\omega}=2 \pi \sqrt{\frac{\gamma m}{\pi} \hbar \omega}\left(\Theta(-\omega) \hat{b}_{-\omega}(0)+\Theta(\omega) \hat{b}_{\omega}^{\dagger}(0)\right)
$$

представляет собой преобразование Фурье силы Ланжевена (20).

Итак, решение уравнения (26) записывается следующим образом:

$$
\tilde{\xi}_{\omega}=\tilde{\xi}_{\omega}^{\mathrm{h}}+\frac{1}{m N(\omega)} \widetilde{F}_{\omega}
$$

где $\tilde{\xi}_{\omega}^{\mathrm{h}}$ представляет собой однородное решение. Обратное преобразование Фурье по времени приводит к выражению

$$
\hat{\xi}(t)=\hat{\xi}^{\mathrm{h}}(t)+\int_{0}^{\infty} d \omega \sqrt{\frac{\gamma}{\pi m} \hbar \omega}\left(\frac{e^{-i \omega t}}{N(-\omega)} \hat{b}_{\omega}+\frac{e^{i \omega t}}{N(\omega)} \hat{b}_{\omega}^{\dagger}\right)
$$

с однородным решением

$$
\hat{\xi}^{\mathrm{h}}(t)=\frac{1}{\sqrt{2 \omega_{1}}}\left(e^{-i \omega_{1} t} \hat{a}+e^{i \omega_{1} t} \hat{a}^{\dagger}\right) e^{-(\gamma / 2) t},
$$

где $\omega_{1}=\sqrt{\Omega^{2}-(\gamma / 2)^{2}}$ и $\left[\hat{a}, \hat{a}^{\dagger}\right]=1$. В данном случае очевиден физический смысл: однородное решение исчезает во времени вследствие затухания, а неоднородное решение осциллятора поддерживается силой Ланжевена.

2.2. Средние значения при тепловом равновесии, свободная энергия и потоки энергии. В термодинамической системе базовые потенциалы представляют собой внутреннюю энергию $E$ и свободную энергию $F$, которые связаны как

$$
E=\frac{\partial}{\partial \beta}(\beta F) .
$$


Далее для рассматриваемой системы необходимо определить средние значения при тепловом равновесии операторов термостата. Хорошо известно, что они следуют из гамильтониана (12) и записываются следующим образом:

$$
\left\langle\hat{b}_{\omega} \hat{b}_{\omega^{\prime}}^{\dagger}+\hat{b}_{\omega}^{\dagger} \hat{b}_{\omega^{\prime}}\right\rangle=\delta\left(\omega-\omega^{\prime}\right) \mathcal{N}_{T}(\omega)
$$

где

$$
\mathcal{N}_{T}(\omega) \equiv \frac{2}{e^{\beta \hbar \omega}-1}+1=\operatorname{cth} \frac{\beta \hbar \omega}{2} .
$$

При наличии наряду с формулами (16) и (30) зависящих от времени решений уравнений движения Гейзенберга (13) и (14) можно исследовать гамильтонианы (3)-(5). Прежде всего обратимся к уравнению $(8)$ и умножим его на $\dot{\hat{q}}_{\omega}(t)$, так что его можно записать в следующем виде:

$$
\frac{d}{d t} \frac{\mu}{2}\left(\dot{\hat{q}}_{\omega}(t)^{2}+\omega^{2} \hat{q}_{\omega}(t)^{2}\right)=\mu \omega^{2} \dot{\hat{q}}_{\omega}(t) \hat{\xi}(t) .
$$

Проведя интегрирование по $\omega$ и используя (4) и (6), получаем

$$
\frac{d}{d t} H_{\mathrm{bath}}=\int_{0}^{\infty} d \omega \mu \omega^{2} \dot{\hat{q}}_{\omega}(t) \hat{\xi}(t)
$$

Аналогичным образом из уравнения (7), путем умножения на $\dot{\hat{\xi}}(t)$ и используя $(3)$ и (6), получаем

$$
\frac{d}{d t} H_{\mathrm{osc}}=\int_{0}^{\infty} d \omega \mu \omega^{2}\left(\hat{q}_{\omega}(t)-\hat{\xi}(t)\right) \dot{\hat{\xi}}(t) .
$$

Затем подставляем решения (16) в (36):

$$
\frac{d}{d t} H_{\text {bath }}=\int_{0}^{\infty} d \omega\left[\dot{\hat{q}}_{\omega}^{\mathrm{h}}(t)+\int_{-\infty}^{t} d t^{\prime} \omega^{2} \cos \left(\omega\left(t-t^{\prime}\right)\right) \hat{\xi}\left(t^{\prime}\right)\right] \hat{\xi}(t) .
$$

Используем (19) и проведем интегрирование по частям:

$$
\frac{d}{d t} H_{\mathrm{bath}}=\dot{F}_{\mathrm{L}}(t) \hat{\xi}(t)+\int_{0}^{\infty} d \omega \mu \omega^{2} \int_{-\infty}^{t} d t^{\prime} \omega \sin \left(\omega\left(t-t^{\prime}\right)\right) \dot{\hat{\xi}}\left(t^{\prime}\right) \hat{\xi}(t) .
$$

Далее аналогичным образом, как при выводе (23), получаем

$$
\frac{d}{d t} H_{\text {bath }}=\left(\dot{F}_{\mathrm{L}}(t)-\gamma m \ddot{\hat{\xi}}(t)\right) \hat{\xi}(t)+\int_{0}^{\infty} d \omega \mu \omega^{2} \dot{\hat{\xi}}(t) \hat{\xi}(t) .
$$

Затем подставляем решение (16) в (37), используем (23) и получаем осциллятор

$$
\frac{d}{d t} H_{\mathrm{osc}}=\left(F_{\mathrm{L}}(t)-\gamma m \dot{\hat{\xi}}(t)\right) \dot{\hat{\xi}}(t) \text {. }
$$

Наконец, при подстановке (16) в (5) и с учетом (19) получаем

$$
H_{\mathrm{int}}=-\left(F_{\mathrm{L}}(t)-m \gamma \dot{\hat{\xi}}(t)\right) \hat{\xi}(t)-\frac{1}{2} \int_{0}^{\infty} d \omega \mu \omega^{2} \hat{\xi}(t)^{2} .
$$

Продолжая, из (40), (41) и (42) видим, что полная энергия сохраняется во времени,

$$
\frac{d}{d t} H=\frac{d}{d t} H_{\mathrm{bath}}+\frac{d}{d t} H_{\mathrm{osc}}+\frac{d}{d t} H_{\mathrm{int}}=0 .
$$

Поскольку отдельные вклады в (43) являются ненулевыми, потоки энергии между ними компенсируют друг друга в любой момент времени. 
2.3. Внутренняя и свободная энергии осциллятора. Используя определения, данные в п. 2.1, вычислим тепловые средние величины гамильтонианов (3)-(5). Поскольку интерес представляет состояние равновесия, введем только неоднородную часть решения (30),

$$
\hat{\xi}(t)=\int_{0}^{\infty} d \omega \sqrt{\frac{\gamma}{\pi m} \hbar \omega}\left(\frac{e^{-i \omega t}}{N(-\omega)} \hat{b}_{\omega}+\frac{e^{i \omega t}}{N(\omega)} \hat{b}_{\omega}^{\dagger}\right) .
$$

Из (43) имеем

$$
\frac{d}{d t} H_{\mathrm{bath}}+\frac{d}{d t} H_{\mathrm{int}}=-\frac{d}{d t} H_{\mathrm{osc}} .
$$

Далее покажем, что соблюдается условие $d\left\langle H_{\mathrm{osc}}\right\rangle / d t=0$. Таким образом, энергия $\left\langle H_{\text {bath }}+H_{\text {int }}\right\rangle=$ const, иными словами, данный параметр не зависит от времени и может быть определен при $t \rightarrow-\infty$, где согласно начальным условиям мы имеем только энергию термостата. Как следствие, интересующая нас внутренняя энергия определяется как

$$
E=\left\langle H_{\mathrm{osc}}\right\rangle
$$

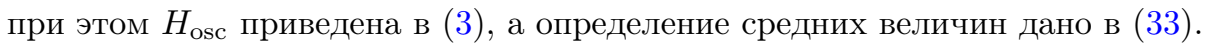

Подставляем решение (44) в (46) и получаем двойное интегрирование по $\omega$, которое при использовании средних величин (33) сводится к однократному интегрированию. Таким образом,

$$
E=\left\langle H_{\mathrm{osc}}\right\rangle=\gamma \int_{0}^{\infty} d \omega \frac{\hbar \omega}{2} \mathcal{N}_{T}(\omega) \frac{\omega^{2}+\Omega^{2}}{|N(\omega)|^{2}},
$$

где использовалось равенство $N(\omega)=N(-\omega)^{*}$. Выражение (47) можно упростить, если использовать следующее тождество:

$$
\gamma \frac{\omega^{2}+\Omega^{2}}{|N(\omega)|^{2}}=\frac{\partial}{\partial \omega} \frac{1}{2 i} \ln \frac{N(\omega)}{N(-\omega)} .
$$

Структура данного выражения позволяет определить фазу:

$$
\delta(\omega)=\frac{1}{2 i} \ln \frac{N(\omega)}{N(-\omega)},
$$

это позволяет записать энергию в виде

$$
E=\int_{0}^{\infty} \frac{d \omega}{2 \pi} \hbar \omega \mathcal{N}_{T}(\omega) \frac{\partial}{\partial \omega} \delta(\omega)
$$

что представляет собой базовую среднюю величину энергии, которая будет использована в дальнейшем. Следует отметить, что средняя величина (47), а также все ей подобные не зависят от времени.

Свободная энергия осциллятора и термостата может быть получена из (50) с использованием (32):

$$
F=T \int_{0}^{\infty} \frac{d \omega}{\pi}\left(\frac{\beta \hbar \omega}{2}+\ln \left(1-e^{-\beta \hbar \omega}\right)\right) \frac{\partial}{\partial \omega} \delta(\omega) .
$$


Интегрирование по частям приводит к иному представлению:

$$
F=-\hbar \int_{0}^{\infty} \frac{d \omega}{2 \pi} \mathcal{N}_{T}(\omega) \delta(\omega)
$$

и, принимая во внимание (34), становится возможным разделить величину свободной энергии на части с нулевой температурой и части, зависящие от температуры:

$$
F=F_{0}+\Delta_{T} F
$$

где

$$
F_{0}=-\hbar \int_{0}^{\infty} \frac{d \omega}{2 \pi} \delta(\omega), \quad \Delta_{T} F=-\hbar \int_{0}^{\infty} \frac{d \omega}{\pi} \frac{1}{e^{\beta \hbar \omega}-1} \delta(\omega) .
$$

Следует отметить, что внутренняя (50) и свободная (51) или (52) энергии выражены через действительные частоты даже в случае диссипации, $\gamma>0$. В целом данные представления не являются новыми. Например, соотношение (47) аналогично выражению (64) из работы [6], а соотношение (50) аналогично выражению (5) из работы [18], где оно названо "замечательной формулой”. Функция $\delta(\omega)(49)$, которую мы называем "фазой”, представляет собой мнимую часть функции Грина в вышеупомянутых работах.

2.4. Частные случаи. Обсудим некоторые частные случаи. Прежде всего, выключим диссипацию, $\gamma \rightarrow 0$. Для этого обратимся к формуле (48) и используем соотношение

$$
\frac{\gamma}{|N(\omega)|^{2}}=\frac{1}{2 i \omega}\left(\frac{1}{N(-\omega)}-\frac{1}{N(\omega)}\right) \underset{\gamma \rightarrow 0}{=} \frac{\pi}{\omega} \delta\left(\omega^{2}-\Omega^{2}\right),
$$

в котором используется теорема Сохоцкого-Племеля, так что формула (48) принимает вид

$$
\frac{\partial}{\partial \omega}\left(\frac{1}{2 i} \ln \frac{N(\omega)}{N(-\omega)}\right) \underset{\gamma \rightarrow 0}{=} \pi \delta(\omega-\Omega) .
$$

В данном случае учитывается условие $\omega \geqslant 0$ при интегрировании (51).

Таким образом, в отсутствие диссипации свободная энергия может быть записана следующим образом:

$$
\left.F\right|_{\gamma=0}=\frac{\hbar \Omega}{2}+T \ln \left(1-e^{-\beta \hbar \Omega}\right)
$$

что представляет собой хорошо известное выражение для одиночного осциллятора в тепловом состоянии.

Далее рассмотрим предел $T \rightarrow 0$ и используем представление (51) для зависящей от температуры части. Таким образом, проведем разложение

$$
\frac{1}{2 i} \ln \frac{N(\omega)}{N(-\omega)}=\gamma \frac{\omega}{\Omega^{2}}+\cdots
$$

для малых $\omega$, что дает в $(54)$ первый член в разложении для $T \rightarrow 0$,

$$
\Delta_{T} F \underset{T \rightarrow 0}{=}-\frac{T^{2} \gamma}{\hbar \Omega^{2}} \frac{\zeta(2)}{\pi}+\cdots
$$


где $\zeta(2)=\pi^{2} / 6$ - дзета-функция Римана. Аналогичная процедура может быть проведена для $\Omega=0$. Используя соотношение

$$
\left.\frac{1}{2 i} \ln \frac{N(\omega)}{N(-\omega)}\right|_{\Omega=0}=\frac{\omega}{\gamma}+\cdots,
$$

получаем

$$
\left.\Delta_{T} F\right|_{\Omega=0} \underset{T \rightarrow 0}{=}-\frac{T^{2}}{\hbar \gamma} \frac{\zeta(2)}{\pi}+\cdots
$$

В обоих случаях для энтропии $S=-\partial F / \partial T$ при $T \rightarrow 0$ имеем

$$
S=\frac{T \gamma}{\hbar \Omega^{2}} \frac{\zeta(2)}{\pi}+\cdots,\left.\quad S\right|_{\Omega=0}=\frac{T}{\hbar \gamma} \frac{\zeta(2)}{\pi}+\cdots .
$$

Следует отметить, что в данном случае не имеет смысла рассматривать предел $\gamma \rightarrow 0$, поскольку это вновь возвращает нас к случаю одиночного осциллятора, как в соотношении (57).

\section{3. ДВА ОСЦИЛЛЯТОРА, ВЗАИМОДЕЙСТВУЮЩИЕ ПОСРЕДСТВОМ ПОЛЯ}

Рассмотрим систему, состоящую из двух осцилляторов, взаимодействующих посредством поля. Лагранжиан записывается как

$$
\begin{aligned}
\mathcal{L}=\int & d x \frac{1}{2}\left(\dot{\phi}(t, x)^{2}-\phi^{\prime}(t, x)^{2}\right)+ \\
& +\sum_{i=1}^{2} \frac{m}{2}\left(\dot{\xi}_{i}(t)^{2}-\Omega^{2} \xi_{i}(t)^{2}\right)-e \sum_{i=1}^{2} \xi_{i}(t) \phi\left(t, a_{i}\right),
\end{aligned}
$$

где $\phi(t, x)$ - поле, $\dot{\phi}$ и $\phi^{\prime}$ - его временна́я и пространственная производные. Осцилляторы описываются их смещениями $\xi_{i}(t)$, собственной частотой $\Omega$ и расположением $a_{i}$. Сила взаимодействия обозначается $e$. Уравнения движения, вытекающие из (63), имеют вид

$$
\begin{aligned}
\left(\partial_{t}^{2}-\partial_{x}^{2}\right) \phi(t, x) & =-e \sum_{i=1}^{2} \xi_{i}(t) \delta\left(x-a_{i}\right), \\
m\left(\partial_{t}^{2}+\Omega^{2}\right) \xi_{i}(t) & =-e \phi\left(t, a_{i}\right), \quad i=1,2 .
\end{aligned}
$$

Данная система напоминает модель плазмы в электродинамике, где диполи (осцилляторы) подчиняются уравнениям движения, аналогичным (64).

В целом система (64) может быть решена двумя способами. В первом случае решаем второе уравнение $(64)$ для $\xi_{i}(t)$ и подставляем результат в первое уравнение, что приводит к эффективному уравнению для поля $\phi(t, x)$. Во втором случае сначала решаем уравнение для $\phi(t, x)$ и подставляем результат во второе уравнение, которое дает эффективное уравнение для $\xi_{i}(t)$.

Первый подход соответствует обычному подходу для рассмотрения сил Казимира-Полдера, а второй подход соответствует некоторому атомистическому подходу. Особенность второго подхода, как мы увидим ниже, в том, что он требует для непротиворечивости поместить поле в конечный ящик. 
Прежде чем обсуждать детали обоих методов, перепишем уравнения (64) в представлении Фурье, используя соглашения (25):

$$
\begin{aligned}
\left(-\omega^{2}-\partial_{x}^{2}\right) \tilde{\phi}_{\omega}(x) & =-e \sum_{i=1}^{2} \tilde{\xi}_{i \omega} \delta\left(x-a_{i}\right), \\
m\left(-\omega^{2}+\Omega^{2}\right) \tilde{\xi}_{i \omega} & =-e \tilde{\phi}_{\omega}\left(a_{i}\right), \quad i=1,2 .
\end{aligned}
$$

Именно эти формулы мы будем использовать в дальнейшем.

3.1. Первый подход. Решим уравнение (66). Его неоднородное решение записывается как

$$
\tilde{\xi}_{i \omega}=-\frac{e}{m N(\omega)} \tilde{\phi}_{\omega}\left(a_{i}\right)
$$

где

$$
N(\omega)=-\omega^{2}+\Omega^{2}
$$

Следует отметить, что его однородное решение определяет вклад в энергию, который не зависит от расстояния $b=a_{2}-a_{1}$ между осцилляторами и который не представляет интерес для нашего дальнейшего рассмотрения. Подстановка (68) в (65) дает

$$
\left(-\omega^{2}-\partial_{x}^{2}-\alpha(\omega) \sum_{i=1}^{2} \delta\left(x-a_{i}\right)\right) \tilde{\phi}_{\omega}(x)=0
$$

- эффективное уравнение для поля, ответственное за взаимодействие с осцилляторами. В данном случае используем представление

$$
\alpha(\omega)=\frac{e^{2}}{m N(\omega)}
$$

которое часто рассматривается как динамическая поляризуемость осцилляторов. Это уравнение подобно приводимому в учебниках уравнению Шредингера с двумя дельта-образными потенциалами. Решение уравнения (69) может быть представлено как

$$
\tilde{\phi}_{\omega}(x)=e^{i \omega x}+\frac{e^{2}}{m} \sum_{r, s=1}^{2} G_{0}^{(\infty)}\left(x-a_{r}\right) \Phi_{r s}^{-1}(\omega) e^{i \omega a_{s}}
$$

где

$$
G_{0}^{(\infty)}(x)=\frac{e^{i \omega|x|}}{-2 i \omega}
$$

- свободная функция Грина, удовлетворяющая условию

$$
\left(-\omega^{2}-\partial_{x}^{2}\right) G_{0}^{(\infty)}(x)=\delta(x)
$$

для $x \in(-\infty, \infty)$, т. е. на всей оси $x$, и предполагается, что $\operatorname{Im} \omega>0$. Введем представление

$$
\Phi_{r s}(\omega)=N(\omega) \delta_{r s}-\frac{e^{2}}{m} G_{0}^{(\infty)}\left(a_{r}-a_{s}\right),
$$

подставим решение (71) в уравнение (69), и, используя (73), можно легко проверить, что $\Phi_{r s}^{-1}(\omega)$ является обратной матрицей $\Phi_{r s}(\omega)(74)$. 
Решение (71) представляет собой решение рассеяния. Его асимптотические разложения имеют вид

$$
\begin{aligned}
& \tilde{\phi}_{\omega}(x) \underset{x \rightarrow-\infty}{\sim} e^{i \omega x}+r(\omega) e^{-i \omega x}, \\
& \tilde{\phi}_{\omega}(x) \underset{x \rightarrow \infty}{\sim} t(\omega) e^{i \omega x},
\end{aligned}
$$

где $r(\omega)$ и $t(\omega)$ - коэффициенты отражения и пропускания. Используя эти формулы, запишем коэффициент пропускания

$$
t(\omega)=1+\frac{e^{2}}{m} \sum_{r, s=1}^{2} \frac{e^{i \omega\left(a_{s}-a_{r}\right)}}{-2 i \omega} \Phi_{r s}^{-1}(\omega) .
$$

Далее, с учетом (74), после проведения алгебраических преобразований выражение (76) может быть переписано в следующем виде:

$$
t(\omega)=\frac{1}{(1+\alpha(\omega) / 2 i \omega)^{2}-\left((\alpha(\omega) / 2 i \omega) e^{i \omega b}\right)^{2}},
$$

где $\alpha(\omega)$ задано в (70), а $b=a_{2}-a_{1}$ - расстояние между осцилляторами.

Теперь, имея выражение для коэффициента пропускания (77), мы располагаем полной информацией, требуемой для получения вакуумной энергии поля $\phi(t, x)$, которая подчиняется уравнению (69). Процедура получения выражения для вакуумной энергии в случае заданного коэффициента пропускания описана во многих публикациях, мы следуем статье [19] (уравнения (14), (15), с представлением $\left.s_{11}(k) \rightarrow t(\omega)\right)$. Определяя фазу рассеяния как

$$
\delta(\omega)=\frac{1}{2 i} \ln \frac{t(\omega)}{t(-\omega)},
$$

получаем формулу для вакуумной энергии

$$
E_{0}=\frac{\hbar}{2} \int_{0}^{\infty} \frac{d \omega}{\pi} \omega^{1-2 \varepsilon} \partial_{\omega} \delta(\omega),
$$

где $\varepsilon>0$ представляет собой ультрафиолетовую регуляризацию, при $\varepsilon \rightarrow 0$ в результате.

Следует отметить, что уравнение (69), помимо решения рассеяния (71), имеет связанные решения, которые задаются полюсами $t(\omega)(77)$ на мнимой оси, скажем при $\omega=i \kappa_{b}$. Показано, что уравнение

$$
t(i \kappa)^{-1}=0
$$

имеет по крайней мере одно решение для всех значений параметров, входящих в (77). Данные связанные решения имеют отрицательную энергию и заселены $\phi$-частицами в основном состоянии системы, образующей некий тип моря Дирака. Следовательно, в данном случае нет необходимости их включения в вакуумную энергию (79).

Представляется удобным провести интегрирование в (79) по мнимым частотам. С этой целью используем (78) и запишем $\delta(\omega)$ как разность двух логарифмов. В первом интеграле поворачиваем интегрирование в положительном направлении, $\omega \rightarrow i \xi$, а во втором интеграле - в отрицательном направлении, $\omega \rightarrow-i \xi$. Эти направления 
заранее заданы выбором $\operatorname{Im} \omega>0$ в (72) и соответствуют повороту Вика. Следуя шагам, описанным в [19], получаем

$$
E_{0}=\frac{\hbar}{2} \cos (\pi \varepsilon) \int_{0}^{\infty} \frac{d \xi}{\pi} \xi \partial_{\xi} \ln |t(i \xi)|+\frac{\sin (\pi \varepsilon)}{2 \pi} \kappa_{b}^{1-2 \varepsilon},
$$

где $\kappa_{b}$ - решение уравнения (80).

Далее можно переписать энергию (81), опуская вклад, который не зависит от расстояния $b$ между осцилляторами, записывая $t(\omega)$ в виде

$$
t(\omega)=\left[1-\left(\frac{\alpha(\omega)}{2 i \omega+\alpha(\omega)} e^{i \omega b}\right)^{2}\right]^{-1} .
$$

Далее, вследствие убывания $\ln |t(i \xi)|$ с $t(\omega)$, заданным уравнением (82) для $\xi \rightarrow \infty$, можно избавиться от регуляризации и положить $\varepsilon=0$. Более того, проведем интегрирование по частям и представим вакуумную энергию в следующем виде:

$$
E_{0}=\frac{\hbar}{2} \int_{0}^{\infty} \frac{d \xi}{\pi} \ln \left|t(i \xi)^{-1}\right|
$$

что в силу (70) записывается в явном виде:

$$
t(i \xi)^{-1}=1-\left(\frac{e^{2} / m}{2 \xi\left(\xi^{2}+\Omega^{2}\right)-e^{2} / m} e^{-\xi b}\right)^{2} .
$$

Соотношения (83), (84) являются формой записи вакуумной энергии, которая известна из силы Казимира-Полдера между осцилляторами. Следует отметить частный случай

$$
E_{0}=-\frac{\pi^{2} \hbar}{24 b}
$$

который получают из (83) при $b \rightarrow \infty$ или $e^{2} \rightarrow \infty$ в том случае, когда задача сводится к задаче на интервале длиной $b$ с граничными условиями Дирихле.

3.2. Второй подход. Начнем с того, что рассмотрим проблему на всей оси. Решение уравнения (65) имеет вид

$$
\tilde{\phi}_{\omega}(x)=-e \sum_{i=1}^{2} G_{0}^{(\infty)}\left(x-a_{i}\right) \tilde{\xi}_{i \omega}
$$

где $G_{0}^{(\infty)}(x)$ определено в $(72)$. Подстановка в (64) дает

$$
\sum_{j=1}^{2}\left(\left(-\omega^{2}+\Omega^{2}\right) \delta_{i j}-\frac{e^{2}}{m} G_{0}^{(\infty)}\left(a_{i}-a_{j}\right)\right) \tilde{\xi}_{j \omega}=0
$$

что является совокупностью алгебраических уравнений. В действительности множитель перед $\tilde{\xi}_{j \omega}$ в $(87)$ представляет собой всего лишь $\Phi_{i j}(\omega)(74)$. Таким образом, собственные частоты (87) заданы нулями $\omega_{s}$ детерминанта:

$$
\operatorname{det} \Phi_{i j}(\omega)=0 \Rightarrow \omega=\omega_{s},
$$


и соответствующая вакуумная энергия просто записывается как

$$
E_{0}=\frac{\hbar}{2} \sum_{s} \omega_{s}^{1-2 \varepsilon}
$$

где $\varepsilon$ - ультрафиолетовая регуляризация.

Однако уравнение $(88)$ с $G_{0}^{(\infty)}(x)$ из $(72)$ не имеет действительных нулей, что легко проверить. Таким образом, выражение (89) не дает действительную вакуумную энергию. Данное обстоятельство уже было описано в работе [20]. Как было отмечено, это происходит потому, что осцилляторы при взаимодействии посредством поля излучают при помещении в бесконечный объем. Для того чтобы избежать этого, помещаем всю систему в конечную клетку (ящик). В данном случае собственные частоты $\omega_{s}$, а вместе с ними и вакуумная энергия (89), становятся действительными. Далее, можно устремить размер клетки (ящика) к бесконечности и рассмотреть зависимость вакуумной энергии от расстояния. Проведем соответствующие вычисления. Реализуем конечный ящик как интервал $x \in[-L / 2, L / 2]$ и поместим осцилляторы в точки $x=a_{1,2} \mathrm{c}$

$$
a_{1,2}=\mp \frac{b}{2},
$$

где $b=a_{2}-a_{1}$ представляет собой расстояние между осцилляторами. На границах поставим условие, что поле $\phi(t, x)$ удовлетворяет граничным условиям Дирихле

$$
\phi\left(t, \pm \frac{L}{2}\right)=0
$$

Необходимо найти функцию Грина $G_{0}(x)$, которая удовлетворяет уравнению (73), как и $G_{0}^{(\infty)}(x)$, и граничным условиям $(91)$. Ее можно построить из базисных функций

$$
\varphi_{n}(x)=\sqrt{\frac{2}{L}} \sin \left(k_{n}\left(x-\frac{L}{2}\right)\right),
$$

где $k_{n}=\pi n / L$. Они образует базис, который удовлетворяет условию

$$
\int_{-L / 2}^{L / 2} d x \varphi_{n}(x) \varphi_{n^{\prime}}(x)=\delta_{n n^{\prime}}
$$

и функция Грина записывается как

$$
G_{0}\left(x, x^{\prime}\right)=\sum_{n=1}^{\infty} \frac{\varphi_{n}(x) \varphi_{n}\left(x^{\prime}\right)}{-\omega^{2}+k_{n}^{2}} .
$$

Можно провести суммирование в (94). Для $|x|<L$ и $\left|x^{\prime}\right|<L$ получаем

$$
G_{0}\left(x, x^{\prime}\right)=\frac{e^{i \omega\left|x-x^{\prime}\right|}-e^{-i \omega\left|x+x^{\prime}\right|}}{-2 i \omega}-\frac{\sin \omega x \sin \omega x^{\prime}}{\omega \sin \omega L} e^{i \omega L} .
$$

Однако нам не требуются соответствующие формулы в общем виде. Отметим два особых случая для формулы (95). Благодаря симметричному расположению осцилляторов (90) эти случаи имеют вид

$$
\begin{aligned}
& G_{0}\left( \pm \frac{b}{2}, \pm \frac{b}{2}\right) \equiv G_{1}(\omega)=\frac{\cos \omega b-\cos \omega L}{2 \omega \sin \omega L} \\
& G_{0}\left(\mp \frac{b}{2}, \pm \frac{b}{2}\right) \equiv G_{2}(\omega)=\frac{1-\cos (\omega(L-|b|))}{2 \omega \sin \omega L}
\end{aligned}
$$


где мы вводим специальные обозначения $G_{1,2}(\omega)$ для этих величин. Отметим, что формулы (96) справедливы для $|b|<L$.

Используя функцию Грина (94), получаем решение уравнения (65), которое подчиняется граничным условиям (91), в следующем виде:

$$
\tilde{\phi}_{\omega}(x)=-e \sum_{i=1}^{2} G_{0}\left(x, a_{i}\right) \tilde{\xi}_{i \omega}
$$

Подставляем решение в уравнение (66) и вместо (87) получаем систему уравнений

$$
\sum_{j=1}^{2}\left(\left(-\omega^{2}+\Omega^{2}\right) \delta_{i j}-\frac{e^{2}}{m} G_{0}\left(a_{i}, a_{j}\right)\right) \tilde{\xi}_{j \omega}=0 .
$$

Снова коэффициенты перед $\tilde{\xi}_{j \omega}$ могут быть записаны в виде $(74)$ как

$$
\Phi_{r s}(\omega)=N(\omega) \delta_{r s}-\frac{e^{2}}{m} G_{0}\left(a_{r}, a_{s}\right) .
$$

Дальнейшая процедура представляется достаточно простой. Определим частоты $\omega_{s}$ как нули

$$
\operatorname{det} \Phi_{i j}(\omega)=0 \Rightarrow \omega=\omega_{s}
$$

детерминанта системы (98) и определим вакуумную энергию, как в (89), но в данном случае частоты $\omega_{s}$ являются действительными и, следовательно, энергия $E_{0}$ действительна. Следует отметить, что действительность $\omega_{s}$ следует из того факта, что мы имеем эрмитов оператор на конечном отрезке.

На следующем этапе рассмотрим вакуумную энергию $E_{0}(89)$ и предел $L \rightarrow \infty$. Для этого перепишем сумму по $s$ в (89) как контурный интеграл, используя утверждение (100) в качестве инструмента, генерирующего моды,

$$
E_{0}=\frac{\hbar}{2} \int_{\gamma} \frac{d \omega}{2 \pi i} \omega^{1-2 \varepsilon} \partial_{\omega} \ln \operatorname{det} \Phi_{i j}(\omega)
$$

где путь $\gamma$ охватывает положительную часть действительной частотной оси. Переписываем логарифм, используя тот факт, что $\Phi_{i j}(\omega)(99)$ представляет собой $(2 \times 2)$-матрицу, как

$$
\begin{aligned}
\ln \operatorname{det} \Phi_{i j}(\omega) & =\operatorname{Tr} \ln \Phi_{i j}(\omega)= \\
& =\ln \left[\left(N(\omega)-\frac{e^{2}}{m} G_{0}\left(a_{1}, a_{1}\right)\right)^{2}-\left(\frac{e^{2}}{m} G_{0}\left(a_{1}, a_{2}\right)\right)^{2}\right] .
\end{aligned}
$$

В соотношении (101) разделим путь интегрирования $\gamma$ на часть $\gamma_{1}$ в верхней полуплоскости $(c \operatorname{Im} \omega>0)$ и часть $\gamma_{2}$ в нижней полуплоскости $(c \operatorname{Im} \omega<0)$ и рассмотрим предел $L \rightarrow \infty$, используя (96). Получаем

$$
\ln \operatorname{det} \Phi_{i j}(\omega) \underset{L \rightarrow \infty}{\sim}\left(N(\omega)-\frac{e^{2}}{m} \frac{1}{-2 i \omega}\right)^{2}-\left(\frac{e^{2}}{m} \frac{e^{i \omega b}}{-2 i \omega}\right)^{2}
$$


на $\gamma_{1}$ и комплексно-сопряженную величину на $\gamma_{2}$. Наконец, повернем путь интегрирования в направлении мнимой оси, $\omega \rightarrow i \xi$ на $\gamma_{1}$ и $\omega \rightarrow-i \xi$ на $\gamma_{2}$, и из (101) получаем

$$
E_{0}=-\frac{\hbar}{2} \cos \pi \varepsilon \int_{0}^{\infty} \frac{d \xi}{\pi} \xi^{1-2 \varepsilon} \partial_{\xi} \ln \left[\left(N(i \xi)-\frac{e^{2} / m}{2 \xi}\right)^{2}-\left(\frac{e^{2} / m}{2 \xi} e^{-\xi b}\right)^{2}\right],
$$

что после проведения преобразований оказывается аналогично соответствующему представлению в п. 3.1. Данное уравнение представляет собой конечную формулу для вакуумной энергии двух диполей, взаимодействующих посредством поля $\phi(t, x)$, для $x$ на всей оси. Напомним, что мы были вынуждены сначала поместить поле $\phi(t, x)$ в конечный ящик, $x \in[-L / 2, L / 2]$. Таким образом, мы получаем действительную вакуумную энергию (101), которая зависит от размера $L$ ящика.

Следует отметить, что для достаточно малого ящика $L<L_{*}$ при

$$
L_{*}=b+\frac{2 m}{e^{2}} \Omega^{2}
$$

не существует действительного решения уравнения (80) и вклад от связанного решения в (81) отсутствует. С увеличением размеров ящика $L$ за пределы $L_{*}$ появляется одно или, в зависимости от параметров $\Omega$ и $b$, два связанных решения. Как уже было отмечено, рассмотрим основное состояние, где они уже заняты. Как будет показано в разделе 4, в данном случае в (104) следует взять модуль выражения в квадратных скобках.

Наконец, сравним (104) и (83). Используя (68), можно показать, что, выделяя из логарифма (104) вклад $\ln \left(N(i \xi)-\left(e^{2} / m\right) / 2 \xi\right)$, который не зависит от расстояния $b$, и затем выкидывая его, мы можем положить $\varepsilon=0$ в (104), поскольку в данном случае логарифм убывает при $\xi \rightarrow \infty$. С учетом модуля и при интегрировании по частям получаем из (104) выражение, аналогичное (83), для вакуумной энергии из п. 3.1.

Таким образом, мы показали, что первый и второй методы, рассмотренные в данном разделе, являются эквивалентными для компоненты вакуумной энергии, зависящей от расстояния. Данная эквивалентность также обсуждается в работе [21] в случае атомистического подхода при выводе взаимодействия ван-дер-Ваальса, а обобщение для запаздывающего случая приведено в работе [20].

\section{4. ДВА ОСЦИЛЛЯТОРА, СОПРЯЖЕННЫЕ С ТЕРМОСТАТОМ И ВЗАИМОДЕЙСТВУЮЩИЕ ПОСРЕДСТВОМ ПОЛЯ}

Рассмотрим полную систему, состоящую из двух осцилляторов, каждый из которых взаимодействует с термостатом и с полем. Такая система, но без термостата, была рассмотрена в разделе 3. Взаимодействие одного осциллятора с термостатом было рассмотрено в разделе 2. В разделе 3 мы были вынуждены рассматривать случай конечного ящика, поскольку иначе не было бы вещественных собственных частот осцилляторов. В настоящем разделе, строго говоря, такого рода мотивация отсутствует, поскольку в состоянии равновесия все возбуждения обеспечиваются силами Ланжевена. Тем не менее мы продолжим рассмотрение системы в конечном ящике. Как будет показано в настоящем и в следующем разделах, переход к ящику большого размера не является тривиальным. Также следует отметить, что на формальном уровне, пока мы работаем в терминах функции Грина $G_{0}\left(x, x^{\prime}\right)$, формулы не меняются. 
Для полной системы лагранжиан имеет вид

$$
\begin{aligned}
\mathcal{L}=\int & d x \frac{1}{2}\left(\dot{\phi}(t, x)^{2}-\phi^{\prime}(t, x)^{2}\right)+\sum_{i=1}^{2} \frac{m}{2}\left(\dot{\xi}_{i}(t)^{2}-\Omega^{2} \xi_{i}(t)^{2}\right)- \\
& -e \sum_{i=1}^{2} \xi_{i}(t) \phi\left(t, a_{i}\right)+\sum_{i=1}^{2} \int_{0}^{\infty} d \omega \frac{\mu}{2}\left(\dot{q}_{i \omega}(t)^{2}+\omega^{2}\left(q_{i \omega}(t)-\xi_{i}(t)\right)^{2}\right),
\end{aligned}
$$

где $\phi(t, x)$ представляет собой поле, а $\dot{\phi}$ и $\phi^{\prime}$ - его временна́я и пространственные производные. Два осциллятора описываются их смещениями $\xi_{i}(t), i=1,2$, а $q_{i \omega}(t)-$ соответствующие переменные термостата. Введем соответствующие канонические импульсы, как это было сделано в разделе 2, и выразим соответствующие части гамильтониана непосредственно через частоты,

$$
\begin{aligned}
H_{\text {field }} & =\int d x \frac{1}{2}\left(\dot{\phi}(t, x)^{2}+\phi^{\prime}(t, x)^{2}\right), \\
H_{\mathrm{osc}} & =\sum_{i=1}^{2} \frac{m}{2}\left(\dot{\xi}_{i}(t)^{2}+\Omega^{2} \xi_{i}(t)^{2}\right) \\
H_{\mathrm{int}} & =e \sum_{i=1}^{2} \xi_{i}(t) \phi\left(t, a_{i}\right) .
\end{aligned}
$$

Кроме того, имеется гамильтониан для полей термостата и их взаимодействия с осцилляторами. Как и в разделе 2, покажем, что данный подход дает только вклад термостата в полную энергию, что не представляет интереса для данного рассмотрения. Таким образом, средние значения при тепловом равновесии величин (107)-(109) дают полную энергию

$$
E=E_{\text {field }}+E_{\text {osc }}+E_{\text {int }}
$$

где

$$
E_{\text {field }}=\left\langle H_{\text {field }}\right\rangle, \quad E_{\text {osc }}=\left\langle H_{\text {osc }}\right\rangle, \quad E_{\text {int }}=\left\langle H_{\text {int }}\right\rangle .
$$

Рассмотрим уравнения движения, которые следуют из лагранжиана (107). Как и в разделе 2 , после устранения полей термостата эти уравнения включают затухание и силы Ланжевена и записывается как

$$
\begin{aligned}
\left(\partial_{t}^{2}-\partial_{x}^{2}\right) \phi(t, x) & =-e \sum_{i=1}^{2} \xi_{i}(t) \delta\left(x-a_{i}\right), \\
m\left(\partial_{t}^{2}+\gamma \partial_{t}+\Omega^{2}\right) \xi_{i}(t) & =-e \phi\left(t, a_{i}\right)+F_{i \mathrm{~L}}(t), \quad i=1,2 .
\end{aligned}
$$

В данном случае

$$
F_{i \mathrm{~L}}(t)=\int_{0}^{\infty} d \omega \frac{\mu \omega^{2} l_{0}}{\sqrt{2}}\left(e^{-i \omega t} \hat{b}_{i \omega}(0)+e^{i \omega t} \hat{b}_{i \omega}^{\dagger}(0)\right)
$$

представляет собой силу Ланжевена для $i$-го осциллятора, а $\hat{b}_{i \omega}(0)$ и $\hat{b}_{i \omega}^{\dagger}(0)$ - операторы уничтожения и рождения для термостата, привязанного к $i$-му осциллятору, которые подчиняются условию

$$
\left[\hat{b}_{i \omega}(0), \hat{b}_{j \omega^{\prime}}^{\dagger}(0)\right]=\delta_{i j} \delta\left(\omega-\omega^{\prime}\right)
$$


а средние значения при тепловом равновесии определяются обобщающим (33) выражением

$$
\left\langle\hat{b}_{i \omega}^{\dagger}(0) \hat{b}_{j \omega^{\prime}}(0)+\hat{b}_{i \omega}(0) \hat{b}_{j \omega^{\prime}}^{\dagger}(0)\right\rangle=\delta_{i j} \delta\left(\omega-\omega^{\prime}\right) \mathcal{N}_{T}(\omega),
$$

где $\mathcal{N}_{T}(\omega)$ задано в $(27)$.

Запишем уравнения (112) после преобразования Фурье (25):

$$
\begin{aligned}
\left(-\omega^{2}-\partial_{x}^{2}\right) \tilde{\phi}_{\omega}(x) & =-e \sum_{i=1}^{2} \tilde{\xi}_{i \omega} \delta\left(x-a_{i}\right), \\
m\left(-\omega^{2}+i \gamma \omega+\Omega^{2}\right) \tilde{\xi}_{i \omega} & =-e \tilde{\phi}_{\omega}\left(a_{i}\right)+\widetilde{F}_{i \omega}, \quad i=1,2,
\end{aligned}
$$

они представляют собой обобщения уравнений (26) и (65), а также (66).

Как и в разделе 3, воспользуемся двумя методами. Следовательно, с самого начала рассмотрим поле $\phi(t, x)$ на конечном отрезке, $x \in[-L / 2, L / 2]$, которое подчиняется граничным условиям Дирихле (91).

4.1. Первый метод. В данном случае решим второе уравнение (116),

$$
\tilde{\xi}_{i \omega}=-\frac{e}{m N(\omega)} \tilde{\phi}_{\omega}\left(a_{i}\right)+\frac{\widetilde{F}_{i \omega}}{m N(\omega)}
$$

получим неоднородное решение в соответствии с процедурой, описанной в разделе 2, и установим, что в присутствии термостата однородное решение исчезает во времени, а возбуждения поддерживаются силами Ланжевена. Затем подставим решение (117) в первое уравнение (116) и получим уравнение

$$
\left(-\omega^{2}-\partial_{x}^{2}-\frac{e^{2}}{m N(\omega)} \sum_{i=1}^{2} \delta\left(x-a_{i}\right)\right) \tilde{\phi}_{\omega}(x)=-\frac{e}{m N(\omega)} \sum_{i=1}^{2} \delta\left(x-a_{i}\right) \widetilde{F}_{i \omega} .
$$

Это уравнение представляет собой неоднородное обобщение уравнения (69). Для решения данного уравнения вводим функцию Грина $G\left(x, x^{\prime}\right)$, которая подчиняется уравнению

$$
\left(-\omega^{2}-\partial_{x}^{2}-\frac{e^{2}}{m N(\omega)} \sum_{i=1}^{2} \delta\left(x-a_{i}\right)\right) G\left(x, x^{\prime}\right)=\delta\left(x-x^{\prime}\right)
$$

и граничным условиям (91). Данная функция Грина может быть представлена в следующем виде:

$$
G\left(x, x^{\prime}\right)=G_{0}\left(x, x^{\prime}\right)+\sum_{r, s=1}^{2} \frac{e^{2}}{m} G_{0}\left(x, a_{r}\right) \Phi_{r s}^{-1}(\omega) G_{0}\left(a_{s}, x^{\prime}\right) .
$$

Здесь свободная функция Грина $G_{0}\left(x, x^{\prime}\right)$ задана в $(94)$ и определено обобщение (99)

$$
\Phi_{r s}(\omega)=N(\omega) \delta_{r s}-\frac{e^{2}}{m} G_{0}\left(a_{r}, a_{s}\right),
$$

где $N(\omega)$ определено в $(27)$ и, как ранее, мы имеем $\sum_{s} \Phi_{r s} \Phi_{s t}^{-1}=\delta_{r t}$. 
Отметим следующий особый случай:

$$
G\left(x, a_{i}\right)=G_{0}\left(x, a_{r}\right)\left(\delta_{r i}+\frac{e^{2}}{m} \Phi_{r s}^{-1}(\omega) G_{0}\left(a_{s}, a_{i}\right)\right)=N(\omega) G_{0}\left(x, a_{r}\right) \Phi_{r s}^{-1}(\omega),
$$

где использовано (121). Для упрощения обозначений в данных формулах и ниже мы используем соглашение о суммировании.

Теперь, используя полученные формулы, мы можем записать решение уравнения (118) как

$$
\tilde{\phi}_{\omega}(x)=-\frac{e}{m N(\omega)} G\left(x, a_{i}\right) \widetilde{F}_{i \omega},
$$

и, используя (122) в более простом виде (при выражении через свободную функцию Грина), получаем

$$
\tilde{\phi}_{\omega}(x)=-\frac{e}{m} G_{0}\left(x, a_{r}\right) \Phi_{r i}^{-1}(\omega) \widetilde{F}_{i \omega} .
$$

Далее подставим (124) в (117),

$$
\tilde{\xi}_{i \omega}=\left(\frac{e^{2}}{m^{2} N(\omega)} G_{0}\left(a_{i}, a_{r}\right) \Phi_{r j}^{-1}(\omega)+\frac{1}{m N(\omega)}\right) \widetilde{F}_{i \omega}
$$

и проведем упрощение с использованием (121),

$$
\tilde{\xi}_{i \omega}=\frac{1}{m} \Phi_{i j}^{-1}(\omega) \widetilde{F}_{j \omega}
$$

Таким образом, формулы (124) и (126) представляют собой решения уравнений движения, полученные первым способом. Оба решения пропорциональны силам Ланжевена.

4.2. Второй метод. В данном случае сначала решим уравнения (116),

$$
\tilde{\phi}_{\omega}(x)=-e \sum_{i=1}^{2} G_{0}\left(x, a_{i}\right) \tilde{\xi}_{i \omega}
$$

где $G_{0}\left(x, x^{\prime}\right)$ задано в (94). Подставим данное решение в уравнения (116) и получим

$$
\left(\left(-\omega^{2}+i \gamma \omega+\Omega^{2}\right) \delta_{i j}-\frac{e^{2}}{m} G_{0}\left(a_{i}, a_{j}\right)\right) \tilde{\xi}_{j \omega}=\frac{1}{m} \widetilde{F}_{i \omega}
$$

где вновь проводим суммирование и с помощью (121), (27) запишем

$$
\Phi_{i j}(\omega) \tilde{\xi}_{j \omega}=\frac{1}{m} \widetilde{F}_{i \omega}
$$

Это алгебраическая система, и ее решение имеет вид

$$
\tilde{\xi}_{i \omega}=\frac{1}{m} \Phi_{i j}^{-1}(\omega) \widetilde{F}_{j \omega}
$$

Наконец, подставим это решение в уравнения (116), что дает

$$
\tilde{\phi}_{\omega}(x)=-\frac{e}{m} G_{0}\left(x, a_{i}\right) \Phi_{i j}^{-1}(\omega) \widetilde{F}_{j \omega} .
$$


Таким образом, формулы (130) и (131) представляют собой решения уравнений движения, полученных вторым способом. Оба решения пропорциональны силам Ланжевена.

Сравнение решений (130) и (131) с решениями (124) и (126) показывает, что оба метода дают одинаковые выражения для решений. Следует отметить, что те же выражения появляются в неоднородных решениях, которые рассматриваются в данном пункте. Уравнения (69) и (87) для однородных решений оказываются различными.

\section{3. Средние значения при тепловом равновесии и свободная энергия.}

Для расчетов свободной энергии последуем процедуре, описанной в разделе 2. Прежде всего рассчитаем средние значения при тепловом равновесии для гамильтонианов (107)-(109) и, следовательно, свободной энергии. Подставляем решения (130) и (131), или аналогично (124) и (126), и получаем средние значения при тепловом равновесии (115). Подстановка решений дает двойные интегралы по $\omega$, и вычисление средних значений при тепловом равновесии устраняет одно из интегрирований. Более того, для осцилляторов появляются двойные суммы, которые аналогичным образом могут быть сведены к однократным суммам. Прежде чем начать данную процедуру, следует отметить, что, используя формулы (25), мы возвращаемся к зависящим от времени решениям.

Процедура вычисления средних значений при тепловом равновесии может быть унифицирована для различных полей. Пусть

$$
\hat{A}(t)=\int_{-\infty}^{\infty} \frac{d \omega}{2 \pi} e^{i \omega t} a(\omega) \widetilde{F}_{\omega}, \quad \hat{B}(t)=\int_{-\infty}^{\infty} \frac{d \omega}{2 \pi} e^{i \omega t} b(\omega) \widetilde{F}_{\omega}
$$

являются двумя операторами подобно решениям (130) и (131). Используя (28), получаем

$$
\begin{aligned}
& \hat{A}(t)=\int_{0}^{\infty} d \omega \sqrt{\frac{\gamma m \hbar \omega}{\pi}}\left(a(-\omega) e^{-i \omega t} \hat{b}_{-\omega}(0)+a(\omega) e^{-i \omega t} \hat{b}_{-\omega}(0)\right), \\
& \hat{B}(t)=\int_{0}^{\infty} d \omega \sqrt{\frac{\gamma m \hbar \omega}{\pi}}\left(b(-\omega) e^{-i \omega t} \hat{b}_{-\omega}(0)+b(\omega) e^{-i \omega t} \hat{b}_{-\omega}(0)\right) .
\end{aligned}
$$

Далее вычислим средние значения при тепловом равновесии симметризуемого произведения данных операторов и, используя (113) и (115), получим

$$
\langle\hat{A}(t) \hat{B}(t)+\hat{B}(t) \hat{A}(t)\rangle=\int_{0}^{\infty} d \omega \frac{\gamma m \hbar \omega}{\pi} \mathcal{N}_{T}(\omega)(a(\omega) b(-\omega)+a(-\omega) b(\omega)) .
$$

Следует отметить, что все операторы в любом случае появляются в соответствующих частях гамильтониана в симметризуемом виде. Как следствие, все средние значения при тепловом равновесии являются действительными. В случае равенства операторов это утверждение является тривиальным.

Более подробно, подставим (124) в (107), используя (111), что дает

$$
E_{\text {field }}=\int_{0}^{\infty} d \omega \frac{\gamma m \hbar \omega}{\pi} \mathcal{N}_{T}(\omega) \frac{e^{2}}{2 m^{2}} \Phi_{i j}^{-1}(\omega) m_{j k} \Phi_{k i}^{-1}(-\omega)
$$

при

$$
m_{j k}=\int_{-L / 2}^{L / 2} d x\left(\omega^{2} G_{0}\left(x, a_{j}\right) G_{0}\left(x, a_{k}\right)+\left(\partial_{x} G_{0}\left(x, a_{j}\right)\right)\left(\partial_{x} G_{0}\left(x, a_{k}\right)\right)\right) .
$$


Далее при подстановке (126) в (108), используя (111), получим

$$
E_{\mathrm{osc}}=\int_{0}^{\infty} d \omega \frac{\gamma m \hbar \omega}{\pi} \mathcal{N}_{T}(\omega) \frac{1}{2 m}\left(\omega^{2}+\Omega^{2}\right) \Phi_{i j}^{-1}(\omega) \Phi_{j i}^{-1}(-\omega)
$$

и, наконец, при подстановке (124) и (126) в (108) получаем

$$
E_{\mathrm{int}}=\int_{0}^{\infty} d \omega \frac{\gamma m \hbar \omega}{\pi} \mathcal{N}_{T}(\omega) \frac{-e^{2}}{m^{2}} \Phi_{i j}^{-1}(\omega) G_{0}\left(a_{j}, a_{k}\right) \Phi_{k i}^{-1}(-\omega)
$$

Отметим, что в данных формулах используется условие суммирования.

Рассмотрим более детально структуру полученных интегралов. Прежде всего имеем равенство

$$
\int_{-L / 2}^{L / 2} d z G_{0}(x, z) G_{0}(y, z)=\frac{1}{2 \omega} \partial_{\omega} G_{0}(x, y)
$$

которое следует из (94) и (93). Далее, имеет место

$$
\int_{-L / 2}^{L / 2} d z\left(\partial_{z} G_{0}(x, z)\right)\left(\partial_{z} G_{0}(z, y)\right)=\left(1+\frac{\omega}{2} \partial_{\omega}\right) G_{0}(x, y)
$$

так что

$$
m_{j k}=\left(1+\omega \partial_{\omega}\right) G_{0}\left(a_{j}, a_{k}\right) .
$$

Просуммируем соответствующие множители в (135), (137), (138):

$$
\begin{aligned}
M \equiv & \frac{e^{2}}{2 m^{2}} \Phi_{i j}^{-1}(\omega) m_{j k} \Phi_{k i}^{-1}(-\omega)+\frac{1}{2 m}\left(\omega^{2}+\Omega^{2}\right) \Phi_{i j}^{-1}(\omega) \Phi_{j i}^{-1}(-\omega)+ \\
& \quad+\frac{-e^{2}}{m^{2}} \Phi_{i j}^{-1}(\omega) G_{0}\left(a_{j}, a_{k}\right) \Phi_{k i}^{-1}(-\omega)= \\
= & \frac{1}{2 m} \Phi_{k i}^{-1}(\omega)\left(\left(\omega^{2}+\Omega^{2}\right) \delta_{i j}+\frac{e^{2}}{m}\left(-1+\omega \partial_{\omega}\right) G_{0}\left(a_{i}, a_{j}\right)\right) \Phi_{j k}^{-1}(-\omega) .
\end{aligned}
$$

Принимая во внимание (121), получим функцию

$$
\Phi_{r s}(\omega)=\left(-\omega^{2}+i \gamma \omega+\Omega^{2}\right) \delta_{r s}-\frac{e^{2}}{m} G_{0}\left(a_{r}, a_{s}\right)
$$

и ее производную

$$
\omega \partial_{\omega} \Phi_{r s}(\omega)=\left(-2 \omega^{2}+i \gamma \omega\right) \delta_{r s}-\frac{e^{2}}{m} \omega \partial_{\omega} G_{0}\left(a_{r}, a_{s}\right) .
$$

Далее составим следующее выражение и упростим его:

$$
\left(\partial_{\omega} \Phi_{i j}(\omega)\right) \Phi_{j k}(-\omega)+(\omega \leftrightarrow-\omega)=2 i \gamma\left(\left(\omega^{2}+\Omega^{2}\right) \delta_{i k}+\frac{e^{2}}{m}\left(-1+\omega \partial_{\omega}\right) G_{0}\left(a_{i}, a_{k}\right)\right) .
$$

Данные формулы позволяют представить (142) в виде

$$
\begin{aligned}
M & =\frac{1}{2 m} \frac{1}{2 i \gamma} \Phi_{k i}^{-1}(\omega)\left[\left(\partial_{\omega} \Phi_{i j}(\omega)\right) \Phi_{j k}(-\omega)+(\omega \leftrightarrow-\omega)\right] \Phi_{k i}^{-1}(-\omega)= \\
& =\frac{1}{2 m} \frac{1}{2 i \gamma}\left[\partial_{\omega} \operatorname{Tr} \ln \hat{\Phi}(\omega)-\partial_{\omega} \operatorname{Tr} \ln \hat{\Phi}(-\omega)\right],
\end{aligned}
$$


где $\hat{\Phi}(\omega)-(2 \times 2)$-матрица с элементами $\Phi_{i j}(\omega)$. Также используем симметрию, следующую из (90) и (96). Правая часть соотношения (146) придает смысл определению

$$
\delta(\omega)=\frac{1}{2 i} \operatorname{Tr} \ln \frac{\hat{\Phi}(\omega)}{\hat{\Phi}(-\omega)},
$$

которое рассматривается вместе с (49) и (78) как некий вид фазы. Следует отметить, что все три выражения различны, а общей является их структура как фаза в комплексном выражении. В данном случае отсутствует неясность, связанная с использованием одного и того же обозначения, поскольку каждое из них применяется только в соответствующем разделе настоящей статьи.

Используя (147), запишем $M$ в окончательном виде:

$$
M=\frac{1}{2 \gamma m} \partial_{\omega} \delta(\omega)
$$

Добавляя энергию (110), получаем из (135), (137), (138) и (142)

$$
E=\int_{0}^{\infty} d \omega \frac{\gamma m \hbar \omega}{\pi} \mathcal{N}_{T}(\omega) M
$$

и с учетом (148)

$$
E=\int_{0}^{\infty} \frac{d \omega}{2 \pi} \hbar \omega \mathcal{N}_{T}(\omega) \partial_{\omega} \delta(\omega)
$$

что представляет собой окончательное выражение для тепловой энергии в системе двух осцилляторов, взаимодействующих с их термостатами и между собой посредством поля. В действительности это выражение похоже на энергию (50) для одиночного осциллятора, взаимодействующего с термостатом, однако смысл фазы $\delta(\omega)$ иной. Более того, формула (150) выглядит как вакуумная энергия (79) поля, взаимодействующего с осцилляторами, где $\delta(\omega)$ имеет смысл сдвига рассеивающей фазы.

Из (150) и (32) получаем соответствующую свободную энергию

$$
F=\int_{0}^{\infty} \frac{d \omega}{\pi}\left(\frac{\hbar \omega}{2}+T \ln \left(1-e^{-\beta \hbar \omega}\right)\right) \partial_{\omega} \delta(\omega)
$$

где очевидно разделение на член с нулевой температурой и часть, зависящую от температуры. Данная формула аналогична формуле (51) в разделе 2. Если выполняется равенство $\delta(0)=0$, проведем интегрирование по частям и получим следующие формулы:

$$
F_{0}=-\frac{\hbar}{2} \int_{0}^{\infty} \frac{d \omega}{\pi} \delta(\omega), \quad \Delta_{T} F=-\hbar \int_{0}^{\infty} d \omega \frac{1}{e^{\beta \hbar \omega}-1} \delta(\omega),
$$

которые также аналогичны соответствующим формулам (54) в разделе 2. Также следует повторить, что при использовании уравнений (150), (151), (152) внутренняя и свободная энергии полной системы, включая диссипацию, выражаются через действительные частоты.

Данный пункт завершим расчетом следа в $\delta(\omega)(147)$. Это возможно, поскольку мы рассматриваем два осциллятора при симметричном расположении (90) внутри 
конечного ящика согласно п. 3.2 и при равной константе связи $e$. В этои случае матрица $\hat{\Phi}(\omega)$ с элементами $\Phi_{r s}(\omega)(143)$ имеет особый вид:

$$
\hat{\Phi}=\left(-\omega^{2}+i \gamma \omega+\Omega^{2}-\frac{e^{2}}{m} G_{1}(\omega)\right) \mathbb{1}-\frac{e^{2}}{m} G_{2}(\omega) \sigma_{1},
$$

где $\mathbb{1}$ представляет собой единичную матрицу и $\sigma_{1}=\left(\begin{array}{ll}0 & 1 \\ 1 & 0\end{array}\right)$, а функции $G_{1,2}(\omega)$ определены в (96). Используя данные формулы, получаем

$$
\operatorname{Tr} \ln \hat{\Phi}(\omega)=\sum_{\sigma= \pm 1} \ln \left(\Phi_{\sigma}(\omega)\right)
$$

где

$$
\Phi_{\sigma}(\omega) \equiv-\omega^{2}+i \gamma \omega+\Omega^{2}-\frac{e^{2}}{m} G_{\sigma}(\omega)
$$

и

$$
G_{\sigma}(\omega) \equiv G_{1}(\omega)+\sigma G_{2}(\omega) .
$$

Соотношение (154) справедливо, поскольку в данном случае матрицы имеют размер $2 \times 2$. Интерпретация такова: значение $\sigma=1$ соответствует симметричной функции $\phi(t, x)$, а $\sigma=-1$ - антисимметричной. Очевидно, что вследствие симметрии энергия представляет собой сумму двух случаев.

Таким образом, вследствие данных формул для $\delta(\omega)(147)$ справедливо представление

$$
\delta(\omega)=\frac{1}{2 i} \sum_{\sigma= \pm 1} \ln \frac{\Phi_{\sigma}(\omega)}{\Phi_{\sigma}(-\omega)},
$$

что мы и будем использовать в дальнейшем.

Выражение (151) - основной результат данного раздела. Для рассматриваемой системы (106) оно представляет собой свободную энергию в случае диссипации в терминах действительных частот. В следующем разделе мы исследуем некоторые свойства такого представления.

\section{5. ЧАСТНЫЕ СЛУЧАИ И ПРЕДСТАВЛЕНИЕ МАЦУБАРЫ ДЛЯ ПОЛНОЙ СИСТЕМЫ В ЯЩИКЕ}

В данном разделе рассмотрим более подробно свойства представления (151) для свободной энергии полной системы (106). Напомним, что данная система помещена в конечный ящик размером $L$.

5.1. Предел $\gamma \rightarrow 0$. Сначала рассмотрим случай $\gamma \rightarrow 0$, иными словами, предел исчезающей диссипации. В выражении для энергии $E(150)$ при $\delta(\omega)$, заданной в (157), нельзя непосредственно положить $\gamma=0$, поскольку нули функций $\phi_{\sigma}(\omega)$ и $\phi_{\sigma}(-\omega)$ оказываются на действительной оси $\omega$ и являются препятствиями на пути интегрирования. Вместо этого применим формулу Сохоцкого-Племеля. Для этого определим

$$
\Phi_{\sigma}^{(0)}(\omega)=\Phi_{\sigma}(\omega)_{\left.\right|_{\gamma=0}}=-\omega^{2}+\Omega^{2}-\frac{e^{2}}{m} G_{\sigma}(\omega) .
$$

Далее отметим, что

$$
\partial_{\omega} \ln \Phi_{\sigma}(\omega)=\frac{\partial_{\omega} \Phi_{\sigma}(\omega)}{\Phi_{\sigma}(\omega)}
$$


Используя указанную формулу, имеем

$$
\frac{1}{\Phi_{\sigma}(\omega)} \underset{\gamma \rightarrow 0}{=}-i \pi \delta\left(\Phi_{\sigma}^{(0)}(\omega)\right)
$$

(главные части сокращаются) и справедливо равенство

$$
\partial_{\omega} \ln \Phi_{\sigma}(\omega)=-i \pi \delta\left(\Phi_{\sigma}^{(0)}(\omega)\right) \partial_{\omega} \Phi_{\sigma}^{(0)}(\omega)
$$

что дает

$$
\partial_{\omega} \delta(\omega)=-\pi \delta\left(\Phi_{\sigma}^{(0)}(\omega)\right) \partial_{\omega} \Phi_{\sigma}^{(0)}(\omega)
$$

Подставляя данное выражение в (150), получаем следующее выражение для энергии:

$$
E \underset{\gamma \rightarrow 0}{=}-\frac{1}{2} \int_{0}^{\infty} d \omega \hbar \omega \mathcal{N}_{T}(\omega) \delta\left(\Phi_{\sigma}^{(0)}(\omega)\right) \partial_{\omega} \Phi_{\sigma}^{(0)}(\omega) .
$$

Функция $\Phi_{\sigma}^{(0)}(\omega)(108)$ описывает систему двух осцилляторов, взаимодействующих посредством поля в отсутствие термостата или при нулевой температуре. Ее нули $\omega_{s}$,

$$
\Phi_{\sigma}^{(0)}\left(\omega_{s}\right)=0,
$$

представляют собой собственные частоты согласно (100), где однако нет разделения на симметричные и антисимметричные решения. Нули уравнения (164) могут быть использованы для представления дельта-функции в (163), в итоге получаем

$$
E=\frac{1}{2} \sum_{s} \hbar \omega_{s} \mathcal{N}_{T}(\omega) \operatorname{sgn}\left(-\partial_{\omega} \Phi_{\sigma}^{(0)}(\omega)_{\mid \omega=\omega_{s}}\right),
$$

что с точностью до знака аналогично (89) (без регуляризации). Появление знаковой функции оказывается немного неожиданным. Однако в данном случае используем простую обходную процедуру. Из (158) получаем

$$
\partial_{\omega} \Phi_{\sigma}^{(0)}(\omega)=-2 \omega-\frac{e^{2}}{m} \partial_{\omega} G_{\sigma}(\omega)
$$

что в точках $\omega=\omega_{s}$ отрицательно, по крайней мере, для малой константы связи, это обеспечивает ожидаемый знак в (165). Таким образом, воспроизводим для $\gamma \rightarrow 0$ представление $E_{0}(89)$, обобщение которого для конечной температуры получено путем выбора $Z=\sum_{s} e^{-\beta \omega_{s}}$. Сходное утверждение справедливо для свободной энергии.

5.2. Предел $T \rightarrow 0$. Рассмотрим предел $T \rightarrow 0$ в зависящей от температуры части $\Delta_{T} F(152)$ свободной энергии. Для этого необходимо получить $\delta(\omega)(157)$ для $\omega \rightarrow 0$. Используя (155), получим

$$
\delta(\omega) \underset{\omega \rightarrow 0}{=} \sum_{\sigma= \pm 1} \frac{\gamma \omega}{\Omega^{2}-e^{2} G_{\sigma}(0)},
$$

что можно использовать вместо (58) и действует аналогично тому, как описано в п. 2.4, тогда имеем

$$
\Delta_{T} F \underset{T \rightarrow 0}{=}-\sum_{\sigma= \pm 1} \frac{\gamma T^{2}}{\hbar\left(\Omega^{2}-\left(e^{2} / m\right) G_{\sigma}(0)\right)} \frac{\zeta(2)}{\pi},
$$

откуда следует знакомый результат для энтропии. 
Из (96) мы получаем

$$
G_{-}(0)=\frac{b(L-b)}{2 L}, \quad G_{+}(0)=\frac{L-b}{2}
$$

и в (167) предполагаем, что $b<L<L_{*}, L_{*}=b+(2 m / b) \Omega^{2}$, т. е. находимся в диапазоне, где знаменатель не меняет свой знак. Более высокие значения $L$ обсудим ниже.

5.3. Переход к представлению Мацубары. Переход к частотам Мацубары для свободной энергии (151) может быть осуществлен при повороте частотного интегрирования по направлению к мнимой оси. Для этого разобьем логарифм в (157) на две части:

$$
\delta(\omega)=\frac{1}{2 i} \sum_{\sigma= \pm 1}\left(\ln \Phi_{\sigma}(\omega)-\ln \Phi_{\sigma}(-\omega)\right) .
$$

Поскольку нули функций $\Phi_{\sigma}(\omega)(155)$ находятся в верхней полуплоскости, повернем путь интегрирования вниз, $\omega \rightarrow-i \xi$. Соответственно второй член направим вверх, $\omega \rightarrow i \xi$. Данный выбор направлений диктуется знаком $\gamma$. Определим функции Грина $(96)$, входящие в $\Phi_{\sigma}(\omega)$, в ящике и продолжим путь интегрирования к обеим сторонам действительной оси $\omega$. Только после перехода к пределу $L \rightarrow \infty$ мы можем определить знак для $\operatorname{Im} \omega$ (в разделе $3 \operatorname{Im} \omega>0$ ). В некотором смысле это можно назвать процедурой "двойного поворота Вика", поскольку поворот только в одном направлении невозможен, и мы должны разбить свободную энергию на две части и осуществить различные повороты.

Далее используем (151),

$$
\frac{\hbar \omega}{2}+T \ln \left(1-e^{-\beta \hbar \omega}\right)=T \ln \left(2 \operatorname{sh} \frac{\beta \hbar \omega}{2}\right)
$$

и его продолжение

$$
\ln \left(2 \operatorname{sh} \frac{\beta \hbar(-i \xi)}{2}\right)=\ln \left|2 \sin \frac{\beta \hbar \xi}{2}\right|-i \pi \sum_{l=0}^{\infty} \Theta\left(\xi-\xi_{l}\right)
$$

где $\xi_{l}=2 \pi T l$ представляет собой частоты Мацубары. Это соотношение вытекает из того, что логарифм имеет разрезы, начиная с $\xi=\xi_{l}$.

Таким образом, получаем

$$
F=T \int_{0}^{\infty} d \xi \sum_{l=0}^{\prime} \Theta\left(\xi-\xi_{l}\right) \partial_{\xi} \ln \Phi_{\sigma}\left(-i \xi_{l}\right)
$$

где мы использовали $\left.\Phi_{\sigma}(\omega)\right|_{\omega=-i \xi}=\left.\Phi_{\sigma}(-\omega)\right|_{\omega=i \xi}$. Таким образом, после поворотов два выражения под знаком суммы в (170) становятся равными.

Наконец, проводим интегрирование по частям и получаем выражение

$$
F=T \sum_{l=0}^{\infty} \sum_{\sigma= \pm 1} \ln \Phi_{\sigma}\left(-i \xi_{l}\right)
$$


для свободной энергии в терминах частот Мацубары. Явные формулы для элементов могут быть записаны следующим образом:

$$
\Phi_{\sigma}\left(-i \xi_{l}\right)=\xi_{l}^{2}+\xi_{l} \gamma+\Omega^{2}-\frac{e^{2}}{m} G_{\sigma}\left(-i \xi_{l}\right)
$$

и из (96) и (158) получаем

$$
G_{\sigma}(-i \xi)=\frac{\operatorname{ch} \xi L-\operatorname{ch} \xi b+\sigma[1-\operatorname{ch}(\xi(L-b))]}{2 \xi \operatorname{sh} \xi L}
$$

Следует отметить, что все приведенные выражения являются действительными, пока размер $L$ ящика невелик, $L<L_{*}$. Также нет сложностей с нулевой частотой Мацубары, как следует из (155) и (169).

\section{6. ЧАСТНЫЕ СЛУЧАИ И ПРЕДСТАВЛЕНИЕ МАЦУБАРЫ ДЛЯ ПОЛНОЙ СИСТЕМЫ ПРИ $L \rightarrow \infty$}

Рассмотрим систему, состоящую из двух осцилляторов, взаимодействующих с полем и с их термостатами на всей оси, иными словами, принимаем предел $L \rightarrow \infty$ в заключительных формулах раздела 4. По-существу ящик учитывается в формулах для свободной энергии (151) свободными функциями Грина $G_{1,2}(\omega)(96)$.

Прежде чем переходить к пределу $L \rightarrow \infty$, отметим, что данные функции Грина включены в выражения для свободной энергии через $\Phi_{\sigma}(\omega)(155)$ и что мы переходим к аналитическому продолжению в нижнюю полуплоскость. Как было показано в п. 5.3, это направление определяется тем обстоятельством, что нули $\Phi_{\sigma}(\omega)$ находятся в верхней полуплоскости. Следовательно, необходимо установить предел в $(96)$ для $\operatorname{Im}(\omega)<0$, что дает

$$
\begin{aligned}
G_{1}^{(\infty)}(\omega) & \equiv \lim _{L \rightarrow \infty} G_{1}(\omega)=\frac{1}{2 i \omega}, \\
G_{2}^{(\infty)}(\omega) & \equiv \lim _{L \rightarrow \infty} G_{2}(\omega)=\frac{e^{-i \omega b}}{2 i \omega} .
\end{aligned}
$$

Подставляя данные выражения в (155), получаем функцию

$$
\Phi_{\sigma}^{(\infty)}(\omega)=-\omega^{2}+i \gamma \omega+\Omega^{2}-\frac{e^{2}}{m} \frac{1+\sigma e^{-i \omega b}}{2 i \omega},
$$

которую следует подставить в фазу $(157)$ вместо $\Phi_{\sigma}(\omega)$, а затем в выражение для свободной энергии (151).

Далее представляет интерес только та часть свободной энергии, которая зависит от расстояния. Это можно получить при записи $\Phi_{\sigma}^{(\infty)}(\omega)$ в виде

$$
\Phi_{\sigma}^{(\infty)}(\omega)=\left(-\omega^{2}+i \gamma \omega+\Omega^{2}-\frac{e^{2}}{2 i \omega m}\right) L_{\sigma}(\omega),
$$

где

$$
L_{\sigma}(\omega)=1-\sigma \frac{\left(e^{2} / m\right) e^{-i \omega b}}{2 i \omega\left(-\omega^{2}+i \gamma \omega+\Omega^{2}\right)-e^{2} / m} .
$$


Первый множитель дает (в виде логарифма) вклад компоненты, которая не зависит от расстояния, и мы опустим его. Второй множитель определяет новую фазу

$$
\delta_{L}(\omega)=\frac{1}{2 i} \ln \frac{L(\omega)}{L(-\omega)}
$$

при

$$
L(\omega) \equiv L_{+}(\omega) L_{-}(\omega)=1-\left(\frac{\left(e^{2} / m\right) e^{-i \omega b}}{2 i \omega\left(-\omega^{2}+i \gamma \omega+\Omega^{2}\right)-e^{2} / m}\right)^{2},
$$

где проводится суммирование по $\sigma$. Таким образом, часть свободной энергии, которая не зависит от расстояния, записывается как

$$
F=\int_{0}^{\infty} \frac{d \omega}{\pi}\left(\frac{\hbar \omega}{2}+T \ln \left(1-e^{-\beta \hbar \omega}\right)\right) \partial_{\omega} \delta_{L}(\omega),
$$

что представляет собой вполне явное выражение.

6.1. Предел $T \rightarrow 0$. Применим подход, как в п. 2.4 или в п. 5.2 , и рассмотрим производную фазы для $\omega \rightarrow 0$

$$
\partial_{\omega} \delta_{L}(\omega)=c_{2}+O\left(\omega^{2}\right)
$$

где

$$
c_{2}=\frac{b^{2}\left(e^{2} / m\right)^{2}+2\left(\gamma-2 b \Omega^{2}\right)\left(e^{2} / m\right)+6 \Omega^{4}}{\left(2 \Omega^{2}-b\left(e^{2} / m\right)\right)\left(e^{2} / m\right)} .
$$

Свободная энергия при $T \rightarrow 0$ записывается как

$$
\Delta_{T} F \underset{T \rightarrow 0}{=}-\frac{T^{2} \zeta(2)}{\pi \hbar} c_{2}+\cdots
$$

Следует отметить, что для $\Omega=0$

$$
\left.c_{2}\right|_{\Omega=0}=-b-\frac{2 \gamma}{b\left(e^{2} / m\right)}
$$

и низкотемпературное поведение $\Delta_{T} F$ не меняется.

6.2. Переход к представлению Мацубары. Переход к частотам Мацубары осуществляется по аналогии с п. 5.3. Разобьем фазу на две части:

$$
\delta_{L}(\omega)=\frac{1}{2 i}(\ln L(\omega)-\ln L(-\omega)),
$$

и повернем контур вниз $(\omega=-i \xi)$ в первой части и вверх $(\omega=i \xi)$ во второй части. Как отмечалось выше, данный подход может рассматриваться как некий вид двойного поворота Вика. Однако, поступая так, мы записываем интеграл по $\omega$ для свободной энергии (182), который является интегралом от разности (187), как разность интегралов. Это нельзя осуществить напрямую, поскольку при $\omega \rightarrow 0$ справедливо

$$
L(\omega)=2 i \omega\left(b-\frac{2 \Omega^{2}}{e^{2} / m}\right)+O\left(\omega^{2}\right),
$$


что следует из (181). Как следствие, каждый из интегралов будет расходиться при $\omega=0$. Очевидное решение - провести интегрирование по $\omega$ сначала с некоторого $\varepsilon>0$ и рассмотреть предел $\varepsilon \rightarrow 0$ после поворотов,

$$
\begin{gathered}
F=\frac{1}{2 \pi i} \lim _{\varepsilon \rightarrow 0}\left[\int_{\varepsilon}^{\infty} d \omega\left(\frac{\hbar \omega}{2}+T \ln \left(1-e^{-\beta \hbar \omega}\right)\right) \partial_{\omega} \ln L(\omega)-\right. \\
\left.-\int_{\varepsilon}^{\infty} d \omega\left(\frac{\hbar \omega}{2}+T \ln \left(1-e^{-\beta \hbar \omega}\right)\right) \partial_{\omega} \ln L(-\omega)\right] .
\end{gathered}
$$

Далее разобьем пути интегрирования на две части. Первая часть представляет собой полукруг радиусом $\varepsilon$, заданный $\omega=\varepsilon e^{-i \varphi}$ в первом интеграле и $\omega=\varepsilon e^{i \varphi}$ во втором интеграле, при этом в обоих случаях $\varphi \in[0, \pi / 2]$. Вторая часть представляет собой прямую линию вдоль мнимой оси при $\omega=-i \xi$ для первого интеграла и $\omega=i \xi$ для второго интеграла, при этом в обоих случаях $\xi \in[\varepsilon, \infty)$. Из интегралов вдоль прямых линий, используя (171), получаем

$$
\begin{aligned}
F_{\text {lin }}= & \frac{T}{2 \pi i} \times \\
& \times\left[\int_{\varepsilon}^{\infty} d \xi\left(\ln \left|2 \sin \frac{\beta \xi}{2}\right|-i \pi \sum_{l=0}^{\infty} \Theta\left(\xi-\xi_{l}\right)\right) \partial_{\xi}\left(\ln |L(-i \xi)|+i \pi \Theta\left(\xi_{*}-\xi\right)\right)-\right. \\
& \left.-\int_{\varepsilon}^{\infty} d \xi\left(\ln \left|2 \sin \frac{\beta \xi}{2}\right|+i \pi \sum_{l=0}^{\infty} \Theta\left(\xi-\xi_{l}\right)\right) \partial_{\xi}\left(\ln |L(-i \xi)|-i \pi \Theta\left(\xi_{*}-\xi\right)\right)\right]
\end{aligned}
$$

В данном случае мы имеем дело с еще одной особенностью, поскольку функция $L(-i \xi)$ имеет ноль, т. е. является действительной при $\xi_{*}>0$ так, что справедливо равенство $L\left(-i \xi_{*}\right)=0$. По этой причине логарифмы $L$ приобретают мнимые части, как показано в приведенных выше формулах. Знаки определяются условием $L(-i \xi)>0$ при достаточно больших $\xi$ и зависят от того, с какой стороны находится точка ветвления логарифма.

Причина появления нулей - связанные состояния, которые обсуждались выше. Также следует отметить, что данная особенность появляется и для конечного ящика при $L>L_{*}$.

Проводим упрощение и интегрирование для вклада при $l=0$ с учетом $\Theta\left(\xi-\xi_{0}\right)=1$ для $\xi>0$; в результате получаем

$$
F_{\text {lin }}=\frac{T}{2} \ln |L(-i \varepsilon)|+T \sum_{l=1}^{\infty} \ln \left|L\left(-i \xi_{l}\right)\right|-T\left|2 \sin \frac{\beta \hbar \xi_{*}}{2}\right| .
$$

В действительности данное выражение расходится при $\varepsilon \rightarrow 0$. При использовании (188) справедливо

$$
\frac{T}{2} \ln |L(-i \varepsilon)|=\frac{T}{2} \ln \varepsilon+\frac{T}{2} \ln \left|2\left(b-\frac{2 \Omega^{2}}{e^{2} / m}\right)\right|+O(\varepsilon),
$$

и расходимость является логарифмической.

Остается рассчитать вклад от полукругов. Ограничивая наше рассмотрение для вкладов, не исчезающих при $\varepsilon \rightarrow 0$, имеем

$$
\frac{\hbar \omega}{2}+T \ln \left(1-e^{-\beta \hbar \omega}\right) \sim \ln \left(\beta \hbar \varepsilon e^{-i \varphi}\right)
$$


для первого вклада и комплексно-сопряженное выражение для второго вклада. Учитывая (188), мы имеем $\partial_{\xi} \ln L(-i \xi)=1 / \xi+O(1)$. Подстановка в (182) дает

$$
\begin{aligned}
F_{\text {h.circ. }} & =-\frac{T}{\pi} \int_{0}^{\pi / 2} d \varphi\left(\ln \left(\beta \hbar \varepsilon e^{-i \varphi}\right)+\ln \left(\beta \hbar \varepsilon e^{i \varphi}\right)\right)= \\
& =-\frac{T}{2} \ln (\beta \varepsilon)+O(\varepsilon) .
\end{aligned}
$$

Мы видим, что расходимость при $\varepsilon \rightarrow 0$, как и следовало ожидать, компенсируется точно такой же расходимостью из (191), и мы получаем сумму выражений (191) и (193) в виде

$$
F=\frac{T}{2} \ln \left|2\left(b-\frac{2 \Omega^{2}}{e^{2} / m}\right) T\right|+T \sum_{l=1}^{\infty} \ln \left|L\left(-i \xi_{l}\right)\right|-T\left|2 \sin \frac{\beta \hbar \xi_{*}}{2}\right| .
$$

Данное выражение и формула

$$
L(-i \xi)=1-\left(\frac{\left(e^{2} / m\right) e^{-\xi b}}{2 \xi\left(\xi^{2}+\gamma \xi+\Omega^{2}\right)-e^{2} / m}\right)^{2}
$$

представляют собой окончательные выражения для свободной энергии в случае диссипации в представлении Мацубары. Разница с формулой (173) может быть сформулирована как формальная замена

$$
L\left(-\left.i \xi\right|_{l=0}\right) \rightarrow 2\left(b-\frac{2 \Omega^{2}}{e^{2} / m}\right) T
$$

для вклада от нулевой частоты Мацубары. Следует отметить, что данная модификация не зависит от диссипации ( $\gamma$ отсутствует в (196)), но скорей является свойством модели.

\section{7. ЗАКЛЮЧЕНИЕ}

В настоящей работе рассмотрена простая система, состоящая из двух гармонических осцилляторов, которые взаимодействуют со скалярным полем размерности $1+1$ и с термостатами, как модель силы Казимира-Полдера в электродинамике. Взаимодействие с термостатами обеспечивает диссипацию, следующую из базовых принципов в том смысле, что данная система имеет гамильтониан. Сначала рассмотрена система без термостата и сделан вывод о том, что в данном случае необходимо сначала использовать конечный ящик для правильного описания. Далее в рассмотрение включен термостат. Выведено относительно простое представление свободной энергии в терминах действительных частот (формулы (151), (157) и (155)), которое отсутствует в литературе, и предложен простой и прямой метод. Заметим, что это еще один пример “замечательной формулы”, описанной в работе [18]. Следует подчеркнуть, что данные действительные частоты не являются собственными частотами (резонансами) диссипативной системы. Исследованы чвстные случаи, такие как исчезающая диссипация, и показано, что результаты воспроизводят случай первоначального отсутствия диссипации.

Более того, рассмотрено низкотемпературное поведение и показано, что термодинамическая проблема отсутствует. Это утверждение также справедливо для случая 
исчезающей собственной частоты $\Omega$ осцилляторов. Рассмотрение проведено для системы в конечном ящике. Далее описан случай $L \rightarrow \infty$, иными словами, случай устранения ящика. Предложена формула для свободной энергии (182) с (180) и (181). В данном случае система всегда обнаруживает особое поведение, что закономерно следует из присутствия дельта-функций в уравнении (69), которые являются притягивающими и приводят к связанным состояниям поля $\phi$ и соответственно к нулям функции $L(-i \xi)$ в формуле (195).

Подобные функции не возникают лишь при большом расстоянии между осцилляторами, что делает разложение асимптотическим. Следует отметить, что особое поведение реализуется и в конечном ящике при условии его достаточно больших размеров.

Интересно отметить, что при увеличении размеров ящика свыше некоторой критической величины в рассматриваемой системе появляются одно или два связанных состояния для поля $\phi$. Мы предполагаем, что соответствующие состояния заселены. В результате в представлении Мацубары появляется дополнительный вклад (последний член в (194)).

Наконец, рассмотрено представление Мацубары для свободной энергии на всей оси. Рассмотрение представления мы начали с представления через действительные частоты и провели некоторый двойной поворот Вика. Получены ожидаемые вклады от частот Мацубары $\xi_{l}$ c $l \geqslant 1$ и, как новый особый случай, проведена модификация вклада от нулевой частоты Мацубары. Показано, что возможна формулировка в рамках формального правила (196). Наряду с представлением свободной энергии в терминах фазы это составляет основной результат настоящей статьи.

Следует отметить, что переход к исчезающей диссипации $(\gamma \rightarrow 0)$ является гладким даже для представления Мацубары. Это следует из соотношения для $L(-i \xi)(195)$, что приводит к аналогичному результату для свободной энергии при $\gamma \rightarrow 0$ при включении $\gamma=0$ с самого начала, как в модели плазмы (уравнение $(84)$ для $\left.t(i \xi)^{-1}\right)$. Причиной такого поведения является присутствие члена $\left(-e^{2} / m\right)$ в знаменателе (195), что может быть связано с присутствием $G_{1}$ в $(176)$ и в $(153)$, это является результатом действия собственного поля осциллятора, конечного в размерности $1+1$. При устранении вклада от собственного поля, что необходимо при переходе к более высоким размерностям [22], предел $\gamma \rightarrow 0$ не воспроизводит свободную энергию в модели плазмы.

Очевидным продолжением настоящей работы является применение методов, предложенных в работе, для силы Казимира-Полдера в электродинамике.

Благодарности. Автор благодарен рецензенту за внимательное прочтение работы.

\section{Список литературы}

[1] V.B. Bezerra, G. L. Klimchitskaya, V.M. Mostepanenko, "Thermodynamical aspects of the Casimir force between real metals at nonzero temperature", Phys. Rev. A, 65:5 (2002), 052113, 7 pp.

[2] G. L. Klimchitskaya, V.M. Mostepanenko, "Conductivity of dielectric and thermal atom-wall interaction", J. Phys. A: Math. Theor., 41:31 (2008), 312002, 9 pp.

[3] G. L. Klimchitskaya, V. M. Mostepanenko, "Casimir free energy and pressure for magnetic metal films", Phys. Rev. B, 94:4 (2016), 045404, 13 pp. 
[4] J. Schwinger, L. L. DeRaad, Jr., K. A. Milton, "Casimir effect in dielectrics", Ann. Phys., 115:1 (1978), 1-23.

[5] D. Kupiszewska, "Casimir effect in absorbing media", Phys. Rev. A, 46:5 (1992), 2286-2294.

[6] F.S. S. Rosa, D. A.R. Dalvit, P.W. Milonni, "Electromagnetic energy, absorption, and Casimir forces: uniform dielectric media in thermal equilibrium", Phys. Rev. A, 81:3 (2010), 033812, 12 pp.

[7] F.S. S. Rosa, D. A.R. Dalvit, P.W. Milonni, "Electromagnetic energy, absorption, and Casimir forces. II. Inhomogeneous dielectric media", Phys. Rev. A, 84:5 (2011), 053813, $13 \mathrm{pp}$.

[8] F. C. Lombardo, F. D. Mazzitelli, A. E. Rubio López, "Casimir force for absorbing media in an open quantum system framework: scalar model", Phys. Rev. A, 84:5 (2011), 052517, $12 \mathrm{pp}$.

[9] P. R. Berman, G. W. Ford, P. W. Milonni, "Nonperturbative calculation of the London-van der Waals interaction potential", Phys. Rev. A, 89:2 (2014), 022127, 4 pp.

[10] М. А. Браун, “Об энергии Казимира в среде с дисперсией и поглощением в рамках подхода диагонализации Фано", ТМФ, 190:2 (2017), 277-292.

[11] H.-P. Breuer, F. Petruccione, The Theory of Open Quantum Systems, Oxford Univ. Press, New York, 2002.

[12] M. Bordag, "Drude model and Lifshitz formula", Eur. Phys. J. C, 71 (2011), 1788, 12 pp.

[13] F. Intravaia, R. Behunin, "Casimir effect as a sum over modes in dissipative systems", Phys. Rev. A, 86:6 (2012), 18 pp.

[14] U. Fano, "Effects of configuration interaction on intensities and phase shifts", Phys. Rev., 124:6 (1961), 1866-1878.

[15] B. Huttner, S. M. Barnett, "Quantization of the electromagnetic field in dielectrics", Phys. Rev. A, 46:7 (1992), 4306-4322.

[16] H. B. Callen, T. A. Welton, "Irreversibility and generalized noise", Phys. Rev., 83:1 (1951), $34-40$.

[17] G. W. Ford, J. T. Lewis, R.F. O'Connell, "Quantum Langevin equation", Phys. Rev. A, 37:11 (1988), 4419-4428.

[18] G. W. Ford, J. T. Lewis, R.F. O'Connell, "Quantum oscillator in a blackbody radiation field", Phys. Rev. Lett., 55:21 (1985), 2273-2276.

[19] M. Bordag, "Vacuum energy in smooth background fields", J. Phys. A: Math. Gen., 28:3 (1995), 755-765.

[20] M. J. Renne, "Retarded Van der Waals interaction in a system of harmonic oscillators", Physica, 53:2 (1971), 193-209.

[21] M. J. Renne, B.R.A. Nijboer, "Microscopic derivation of macroscopic Van der Waals forces", Chem. Phys. Lett., 1:8 (1967), 317-320.

[22] M. Bordag, J. M. Muñoz-Castañeda, "Dirac lattices, zero-range potentials and self-adjoint extension", Phys. Rev. D, 91:6 (2015), 065027, 19 pp.

Поступила в редакцию 31.05.2017, после доработки 16.07.2017 